Interfaces

\title{
De la page blanche à la sculpture, les multiples formes de l'objet-livre - Entretien
}

\section{Alexandre Melay et Valérie Morisson}

\section{(2) OpenEdition}

1 Journals

Édition électronique

URL : https://journals.openedition.org/interfaces/2875

DOI : 10.4000/interfaces.2875

ISSN : 2647-6754

Éditeur :

Université de Bourgogne, Université de Paris, College of the Holy Cross

\section{Édition imprimée}

Date de publication : 12 juillet 2021

ISSN : 1164-6225

\section{Référence électronique}

Alexandre Melay et Valérie Morisson, « De la page blanche à la sculpture, les multiples formes de l'objet-livre - Entretien », Interfaces [En ligne], 45 | 2021, mis en ligne le 12 juillet 2021, consulté le 16 septembre 2021. URL : http://journals.openedition.org/interfaces/2875 ; DOI : https://doi.org/ 10.4000/interfaces.2875

Ce document a été généré automatiquement le 16 septembre 2021.

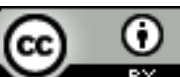

Les contenus de la revue Interfaces sont mis à disposition selon les termes de la Licence Creative Commons Attribution 4.0 International. 


\title{
De la page blanche à la sculpture, les multiples formes de l'objet-livre - Entretien
}

\author{
Alexandre Melay et Valérie Morisson
}

Figure 1. Inventaire personnel de différents formats de livres d'artiste. 2011-2017.

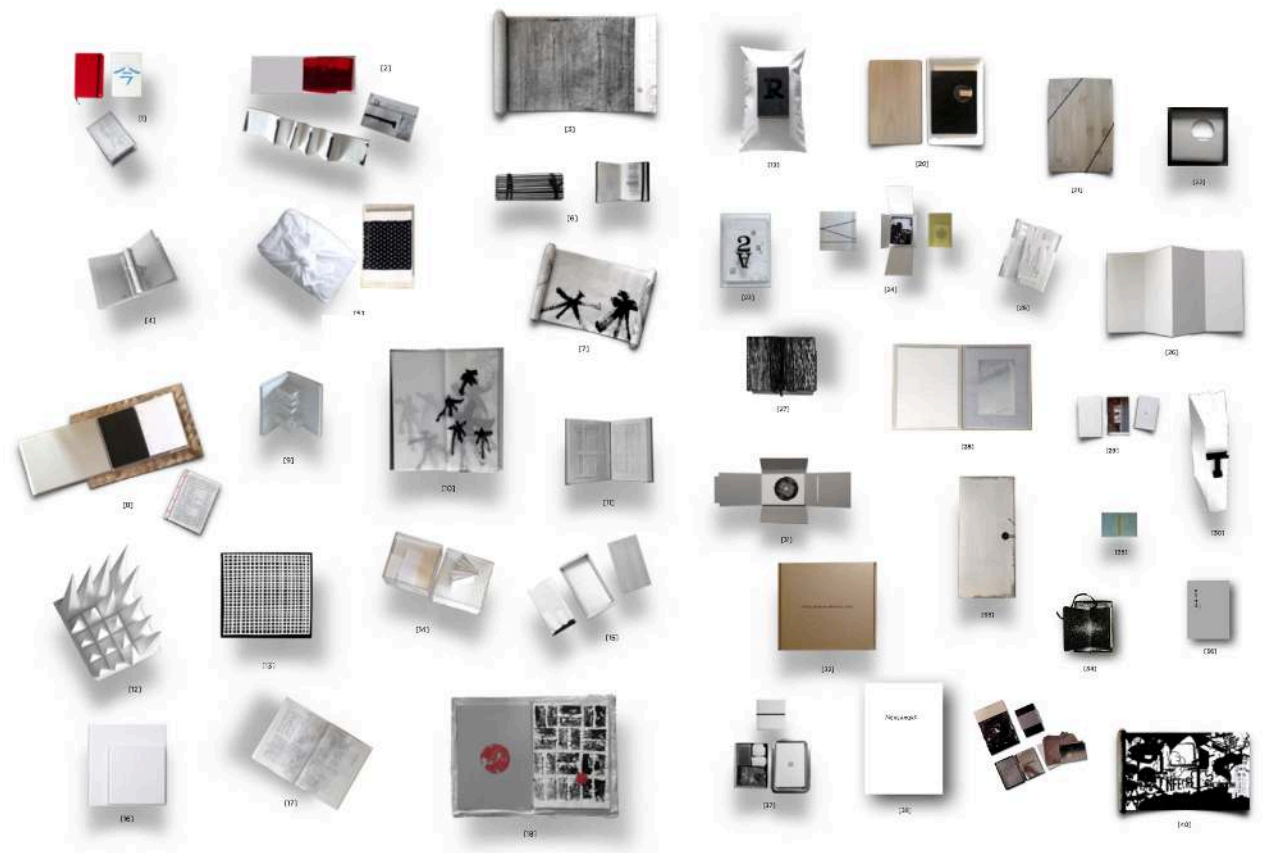

(c) Alexandre Melay.

VALÉRIE MORISSON : Vous avez beaucoup travaillé sur le livre et proposé de nombreuses variations autour de sa forme et de son format. Je suis donc ravie que vous ayez accepté de nous présenter certaines de vos œuvres dans le contexte de ce volume. Au cours du XXe siècle, plusieurs artistes se sont attachés à déconstruire le paradigme du livre carré ou rectangulaire et ont proposé des formes expérimentales qui interrogent la relation entre le 
livre et son contenu. Dans votre propre pratique, c'est la question du format et des rencontres entre les éléments graphiques, plastiques, sculpturaux, voire architecturaux qui vous inspire. Vous parlez d'objets-livres d'ailleurs.

Alexandre Melay : J'utilise en effet le terme d'"objet-livre" car je considère le livre d'artiste comme un objet "autre", avec ses formes singulières et étranges; il n'est ni tout-à-fait livre ni tout-à-fait objet. L'objet-livre est un livre qui attire l'attention sur lui-même par sa forme insolite ou la matière dans laquelle il est réalisé. J'utilise l'objet-livre comme un médium, un véritable territoire, le terrain d'une activité empirique : l'"espace du livre" devient dès lors un espace producteur de sens où se joue un dialogue étroit entre support, forme et contenu. Ce sont les premiers objetslivres contemporains sous une forme différente, imaginés par Man Ray ou Marcel Duchamp (La Boite verte, 1934), qui ont incité de nombreux artistes contemporains à concevoir des objets-livres atypiques - on pense notamment à Michel Butorentraînant la production de multiples étonnants qui ont permis d'instaurer une nouvelle façon de réfléchir, en tant qu'artiste, sur la production de livre. Le livre, depuis les années 1960, est devenu un objet artistique expérimental, pouvant même se métamorphoser parfois en espace d'exposition. C'est grâce à son utilisation en tant que médium que le livre a acquis une personnalité matérielle et formelle, en devenant "objet-livre" où la page se transforme en une sculpture transmettant de l'information. Et ce sont bien les techniques graphiques et plastiques, appliquées au livre, qui engendrent des formes et des formats inédits. En fin de compte, le pari du livre d'artiste est de ré-enchanter la matérialité livresque, de faire émerger un espace et un sens nouveaux en composant avec le livre de manière à renouveler ses formes, et à ainsi le "ré-inquiéter".

Mes réalisations livresques font référence à l'architecture et entretiennent une filiation avec l'art minimal et conceptuel dans une esthétique japonisante. Ces livres d'artiste jouent avec le papier, la photographie, le signe. Ils sont à comprendre comme des formes conceptuelles qui reprennent certains codes et esthétiques pour proposer un objet hybride, un assemblage de plusieurs éléments, qu'ils soient graphiques, plastiques, photographiques ou textuels. Je trouve très intéressant de concevoir l'élaboration du livre comme une "construction" au sens noble du terme. En effet, le rapport entre l'architecture et le livre est pour moi très important ; j'aime construire un livre comme un architecte construit un bâtiment. Car le livre est toujours tridimensionnel et se déploie comme un édifice en papier. Pour moi, le livre est comme l'architecture : il se construit étape par étape. Et c'est bien la question du format, de l'aspect formel qui est au centre de ma production. Cette dernière est ellemême à comprendre comme une expérimentation où chaque objet devient une "forme conceptuelle".

vM : Quand vous parlez de ré-inquiéter le livre dans son format, de jouer avec les normes et les cadres formels, intellectuels et interprétatifs, je ne peux m'empêcher de faire un parallèle avec d'autres libertés prises avec le cadre puisque de nombreux peintres ont fait voler en éclat le cadre entourant la toile et, parfois, le châssis. Quelles matrices revisitezvous ? Quels livres représenteraient la norme à transgresser?

AM : De la page blanche à la sculpture, du livre miniature au format XXL, les différentes variations du format de l'objet-livre se multiplient, créant une rupture avec la géométrie du codex, avec ce que Michel Melot nomme "l'empire du cadre » dans Livro (2012). Le travail sur le « corps du livre », le «physique du livre ", comme le définit Michel Butor, me permet de créer une diversité de formats. Car mes 
différents objets-livres s'inscrivent tous dans un "système" varié de formats, entre forme et norme. Dans mes livres d'artiste, alors que le concept de format évoque habituellement des notions et des contraintes, un cadre et une grille, il aboutit à une transgression de la géométrie du codex et des formes hybrides : absence de couverture, de cahiers, de pages numérotées... Cette transgression du support bidimensionnel me permet de rendre l'objet-livre proche de la sculpture ou de l'architecture. C'est un objet en trois dimensions, sculptural, qui prend des formes aussi variées qu'inattendues : des "systèmes" de formats que l'on peut appeler "livreaccordéon", "livre-maquette", "livre-rouleau", "livre-carnet", "livre-édifice", "livrecarte", "livre-emboîtage", "livre-planche", ou encore "livre enveloppé" ou "livre sonore".

VM : Beaucoup de vos œuvres font référence à la culture japonaise. À quel moment vous y êtes-vous intéressé ? Y a-t-il au Japon un imaginaire et une pratique du livre très différente qui invite à une autre relation à l'objet?

$\mathrm{AM}$ : Il y a, en effet, une culture du livre et de l'imprimé très présente au Japon, et l'étude du livre, de l'écrit et de l'image y a connu des développements considérables. Il existe un véritable culte de ce que l'on appelle le book design, une histoire de l'imprimé parfaitement singulière que l'on retrouve dans les livres d'artiste, les livres d'estampes et une culture du papier, du signe, du graphisme importante, une passion prononcée pour le visuel. Dans la culture japonaise, l'impact d'un livre ou d'une revue l'emporte nettement sur l'exposition de quelques images. L'origine idéographique de l'écriture japonaise donne aussi une centralité à l'image qu'elle n'a pas dans nos cultures occidentales. L'importance du graphisme, de la mise en page de l'image était déjà centrale dans le phénomène de l'estampe. Tout ceci fait de ce pays une civilisation du visuel, du signe et de l'image.

Le temps semble déjà bien loin, lorsque durant mon enfance, je découvris les représentations majestueuses de la montagne la plus sacrée et la plus vénérée du Japon, le Fuji-San, réalisées par le maître de l'estampe Hokusai Katsushika. Mais l'expérience japonaise à proprement parler est arrivée bien plus tard. Je ne saurais expliquer précisément pourquoi mais la culture japonaise me captiva et marqua profondément mon esprit, nourrissant mon imagination et contribuant à stimuler intellectuellement et artistiquement ma démarche. J'ai découvert le "signe", ce signe japonais présent dans l'ensemble de la culture au Japon et dont Roland Barthes parle dans L'Empire des signes (1970). Je me suis découvert une profonde affinité avec la rigueur géométrique de cette civilisation, en m'ouvrant à un pays à la puissance architecturale, esthétique et spirituelle. C'est aussi lors de ces séjours que j'ai visité et étudié les structures de différents temples et jardins, tout en collectant livres de calligraphies, objets, céramiques, "matériaux japonais". Tout un vocabulaire abstrait et complexe aux motifs géométriques qui ont le pouvoir d'être des outils ou des matériaux pour la conception de mes futurs livres. Mon art du livre est intimement lié à mes séjours au Japon, à ce que j'appelle une parenthèse japonaise. Car la culture japonaise a un rapport avec les arts graphiques. Je ressens l'esthétique japonaise comme révélatrice, productrice de quelque chose de raffiné, de protégé, un ailleurs, une parenthèse de délicatesse, de repos, de Beau. Le Japon est le temps des choses belles, de la photographie, des artisans d'art, de la tradition, du passé, de l'artisanat... La pratique de la calligraphie, la découverte de l'estampe ainsi que du livre photographique japonais m'ont aussi permis de réaliser de nombreux livres photographiques et autres objets-livres, entre abstraction et figuration, signes et 
motifs, marqués par une approche minimaliste dépouillée, souvent en noir et blanc, reflétant l'esthétique du vide, l'atemporalité, la nostalgie propres à ce pays, mais également le design graphique japonais moderne et contemporain.

vм: Certains de vos objets-livres conservent le format du carnet, un objet qui a accompagné de nombreux artistes et où l'on consigne des impressions, des observations faites sur le vif. Le carnet a quelque chose d'intime et d'inchoatif. Quels sont les éléments classiques du carnet que vous préservez et quels sont ceux que vous transformez?

AM : Tout à fait, le carnet est très important dans ma pratique personnelle ; j'utilise le carnet comme une zone de pensée, permettant de conserver des mots, des dessins, des croquis, des informations, de répertorier, au sein d'un même objet, une multiplicité d'éléments pour ensuite les utiliser a posteriori. C'est souvent le point de départ pour la création de livres. Pour ce qui est du format carnet, digne héritier de son format originel, les objets-livres de ce type permettent, comme c'est le cas dans Book of dreams (2010), de devenir un support où viennent se poser pensées et souvenirs de voyage, un récit textuel et graphique composé de techniques mixtes, de dessins et d'écritures réalisés à l'encre ou à la peinture acrylique, au crayon, au stylo et mélangés avec différents collages, dans une tonalité de noir et blanc jouant sur les rapports entre les pleins et les vides, le clair et le sombre (Fig. 2). L'objet-livre prend sa forme définitive lorsque le carnet est enfermé dans sa boîte noire, lui servant aussi de support de présentation.

Figure 2. Book of dreams, dessins et textes, techniques mixtes, encre, acrylique, crayon, stylo, collage, noir \& blanc, boîte noire et tirages photographiques noir et blanc, $90 \times 140$ mm, 2010 .
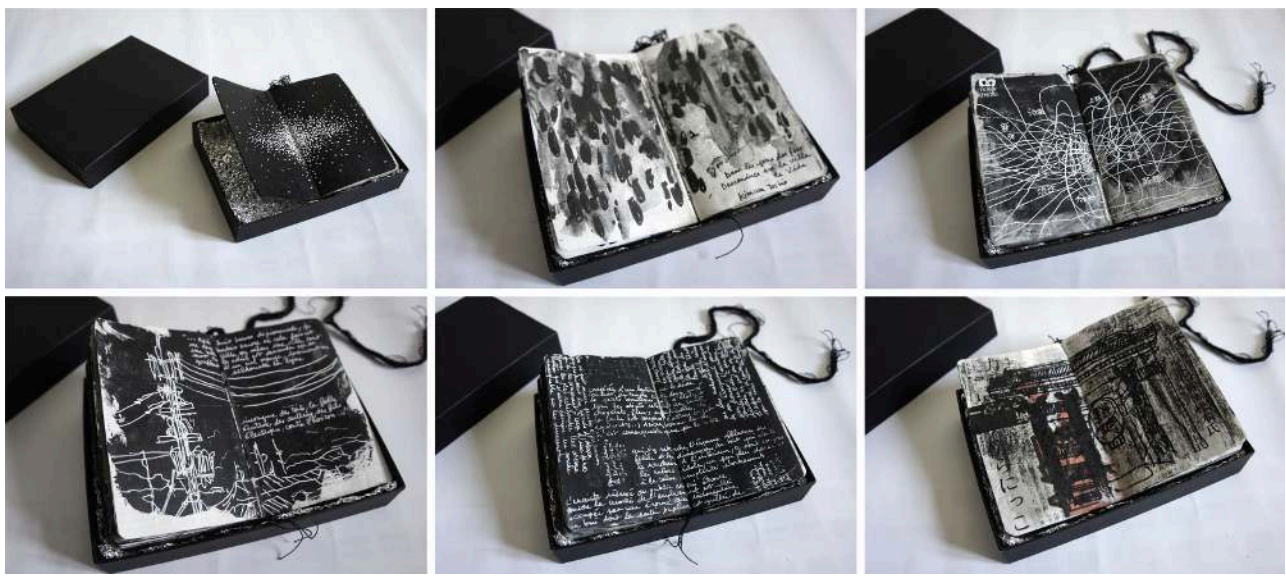

(c) Alexandre Melay.

Dans un autre objet-livre intitulé Phenomenon (2012), réalisé à partir des pages déchirées d'un carnet, j'ai cherché à donner au papier une apparence qui rappelle les troncs des arbres qui, ensemble, forment une forêt (Fig. 3). La couleur noire m'a été inspirée par la technique japonaise du bois brûlé. En réalisant l'objet-livre dans un carnet, j'ai souhaité que les déchirures asymétriques et les nuances de noirs et de gris de la peinture lui donnent une touche sensible et visuelle. Mais le livre demeure énigmatique et sa présentation verticale interroge le lecteur : il est transporté dans un voyage intérieur qui suscite l'émotion, notamment par l'essence de bois d'hinoki, reconnu pour ses effets thérapeutiques, qui imprègne les pages du carnet; un livre olfactif qui renvoie à la forêt et à ses odeurs. 
Figure 3. Phenomenon, peinture acrylique sur feuilles de papier, déchirures, boîte en bois, $160 \times 225$ $\mathrm{mm}, 2012$.

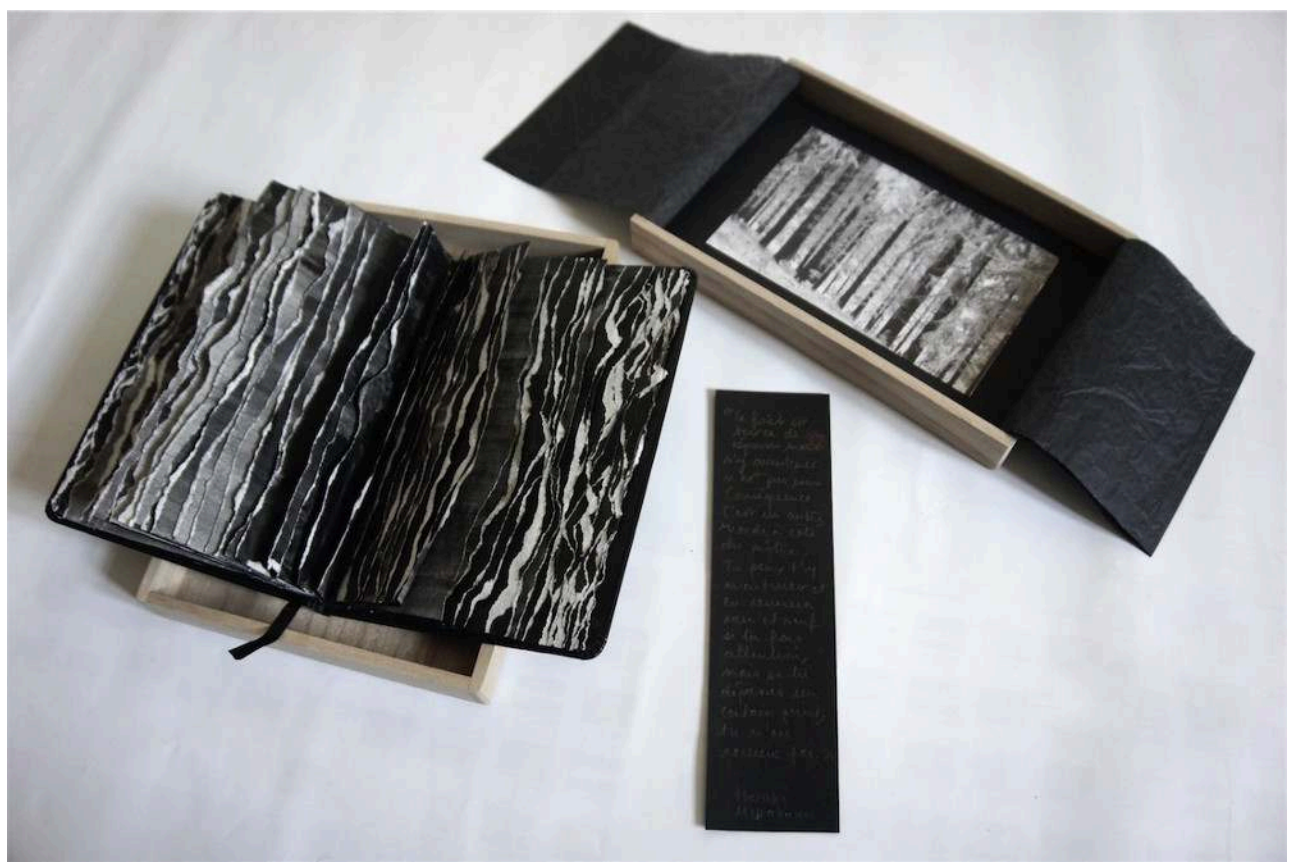

(c) Alexandre Melay.

Dans une autre approche du carnet, j'ai réalisé Kyoto [Layers and Patterns] Architecture (2014) en réutilisant notamment les caractéristiques du carnet japonais, à savoir la reliure traditionnelle très spécifique qui ne nécessite pas de colle mais seulement du fil et une aiguille permettant de relier les feuilles entre elles, ainsi que les proportions japonaises du format B5 (soit 176 x $250 \mathrm{~mm}$ ). De même, j'ai voulu des couvertures dotées de propriétés tactiles, lisses ou rugueuses, qui invitent au toucher, à la manipulation. Les deux carnets, dont les pages sont par alternance blanches et translucides, sont composés de textes, de dessins et de photographies. À l'intérieur, chaque "livre-carnet" prend la forme d'un inventaire des textures et des motifs de l'architecture traditionnelle, des éléments, des structures qui peuvent servir de modèle à la conception architecturale contemporaine (Fig. 4).

Figure 4. Kyoto [Layers and Patterns] Architecture, série de deux ouvrages, impression numérique noir \& blanc, reliure japonaise ; textes, dessins et photographie insérés, dimensions variables, 2014.
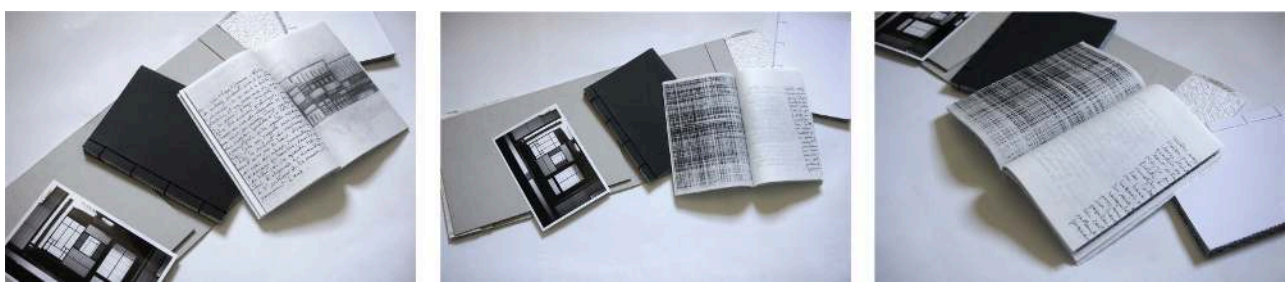

(C) Alexandre Melay.

vм : Un autre format traditionnel, d'origine japonaise, que vous réinterprétez est le rouleau. Le rouleau conserve la linéarité et la narrativité du livre. Néanmoins, il peut aussi évoquer les cartes ou les dessins d'architectes enroulés. II faut, dans ces différents cas, dérouler le 
support et se laisser aller à une herméneutique particulière. Comment analysez-vous le déploiement induit par ce format?

AM : Dans le livre d'artiste, le rouleau demeure en effet un format très prisé. C'est une forme traditionnelle qui a longtemps été utilisée par la culture japonaise, et notamment dans la calligraphie ; un format horizontal, qui rappelle les emakimono, cette peinture japonaise consistant en une alternance d'extraits de romans calligraphiés et d'illustrations, qui assurent, ensemble, le récit d'une histoire que l'on découvre au fur et à mesure que se déroule le rouleau, de droite à gauche et par bandes de $60 \mathrm{~cm}$. Grâce à ce format, le regard latéral qui balaie le livre instaure un parcours narratif. C'est un principe que j'ai réutilisé dans l'œuvre Shades of space (2014) à partir d'une peinture sur papier calque dépliable et enroulée sur un rouleau en bois (Fig. 5). Comme une carte qui se déroule, faite de multiples points, de zones plus ou moins denses, de gradations, de nuances de noirs, de gris et de blancs : le déroulé d'une texture, d'une matière. Cette forme en rouleau implique que l'objetlivre soit expérimenté, ouvert, dévoilé, manipulé. Elle suppose des déploiements multiples et un mouvement contraint par l'espace et le temps puisque l'objet-livre n'est déroulé que sur une distance de $60 \mathrm{~cm}$ pour respecter la tradition japonaise.

Figure 5. Shades of space, peinture acrylique sur papier calque, rouleau, dimensions variables, 2014.
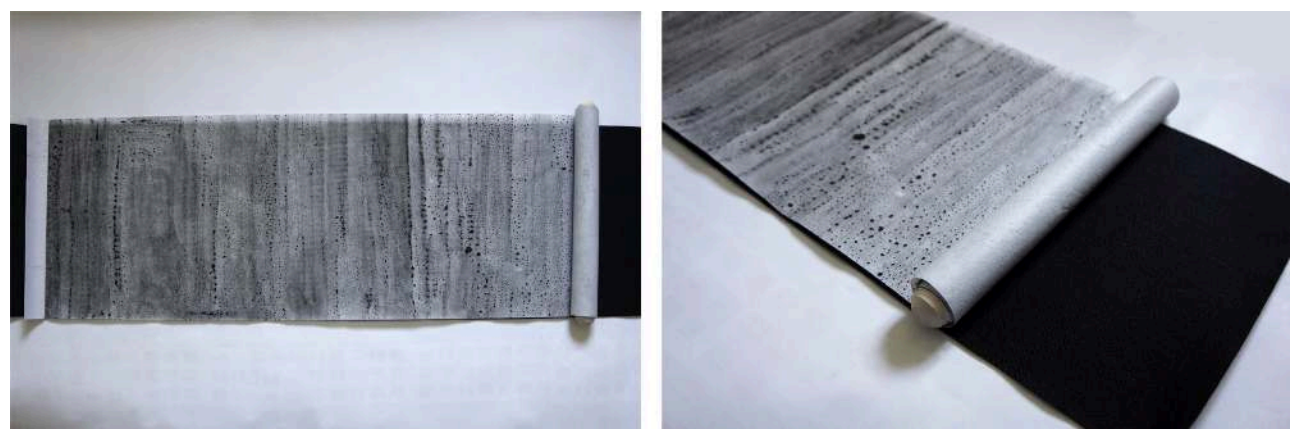

(c) Alexandre Melay.

Figure 6. Black \& white flux, peinture sur papier calque, polystyrène, rouleau, $305 \mathrm{~mm} ; 320$ x 450 $\mathrm{mm}, 2017$.
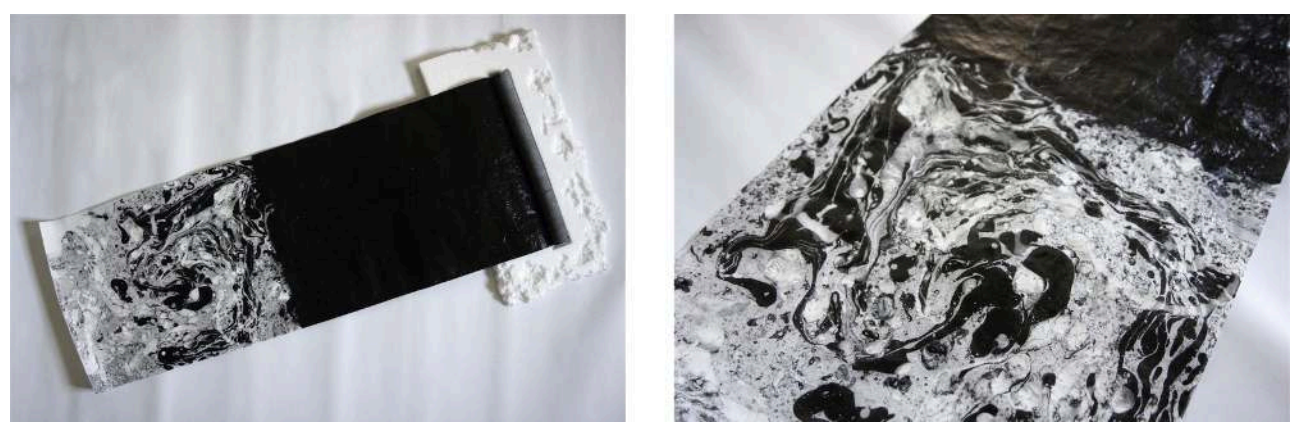

(c) Alexandre Melay.

Ce même système de déroulement-enroulement est utilisé dans Black \& white flux (2017), un dispositif qui permet de retranscrire l'image du flux de l'eau et du mouvant rendu possible grâce une longueur de papier presque infinie (Fig. 6). Dans ce livre, le format linéaire me permet de montrer une progression dans le surgissement de la peinture réalisée dans deux couleurs: le blanc du début va se mélanger au fil du 
déroulement et former une zone aqueuse presque fluide pour finir sur un noir profond. Et c'est le déroulement complet de l'objet qui va dévoiler un haiku (un court poème japonais non rimé caractérisé par sa forme extrêmement brève et son format très restreint limité à dix-sept syllabes) de l'auteur Masaoka Shiki : Au long de la rivière - je n'ai vu aucun pont - ce jour est sans fin.

VM : Le rouleau et le livre-accordéon partagent-ils beaucoup de caractéristiques ?

AM : D'une certaine manière, le système du rouleau s'apparente à la forme en accordéon ou « leporello » qui est une autre forme traditionnelle du livre d'artiste et qui permet également un déploiement dans l'espace, en renvoyant notamment au cinéma. Cet autre format linéaire joue un rôle important dans la restitution de ce caractère spatial. Dans le livre d'artiste Invisible Cities (2012), le point de départ a été l'ouvrage d'Italo Calvino, Les villes invisibles (Invisible Cities) et j'ai tout de suite pensé à ce système de plis (Fig. 7). La pliure créée par le système accordéon que l'on retrouve aussi dans l'objet-livre Transparence (2015) (Fig. 8). Toute en transparence, la forme de cet objet s'apparente à un livre dont on aurait détaché les pages une par une. Sur les différentes pages transparentes non reliées, j'ai gravé minutieusement la retranscription du célèbre texte bouddhiste, le Hannya-Shingyo (Sutra du Cœur). Cette présentation particulière permet aux pages qui se superposent de créer un effet de strates infinies dans lequel le regard se perd. L'objet-livre, dans son ensemble, permet de jouer sur un effet de transparence, tel un dispositif optique laissant percer la lumière comme un effet de vitrail. La transparence de l'écriture emprisonne le temps, l'éternité. Ici, il est question de transcendance, ce qui implique l'idée de dépassement ou de franchissement, de surpassement, ce qui est au-delà du perceptible, ce qui dépasse ou surpasse les possibilités de l'entendement.

Figure 7. Invisible cities. Livre-accordéon blanc, dimensions variables. 2012.

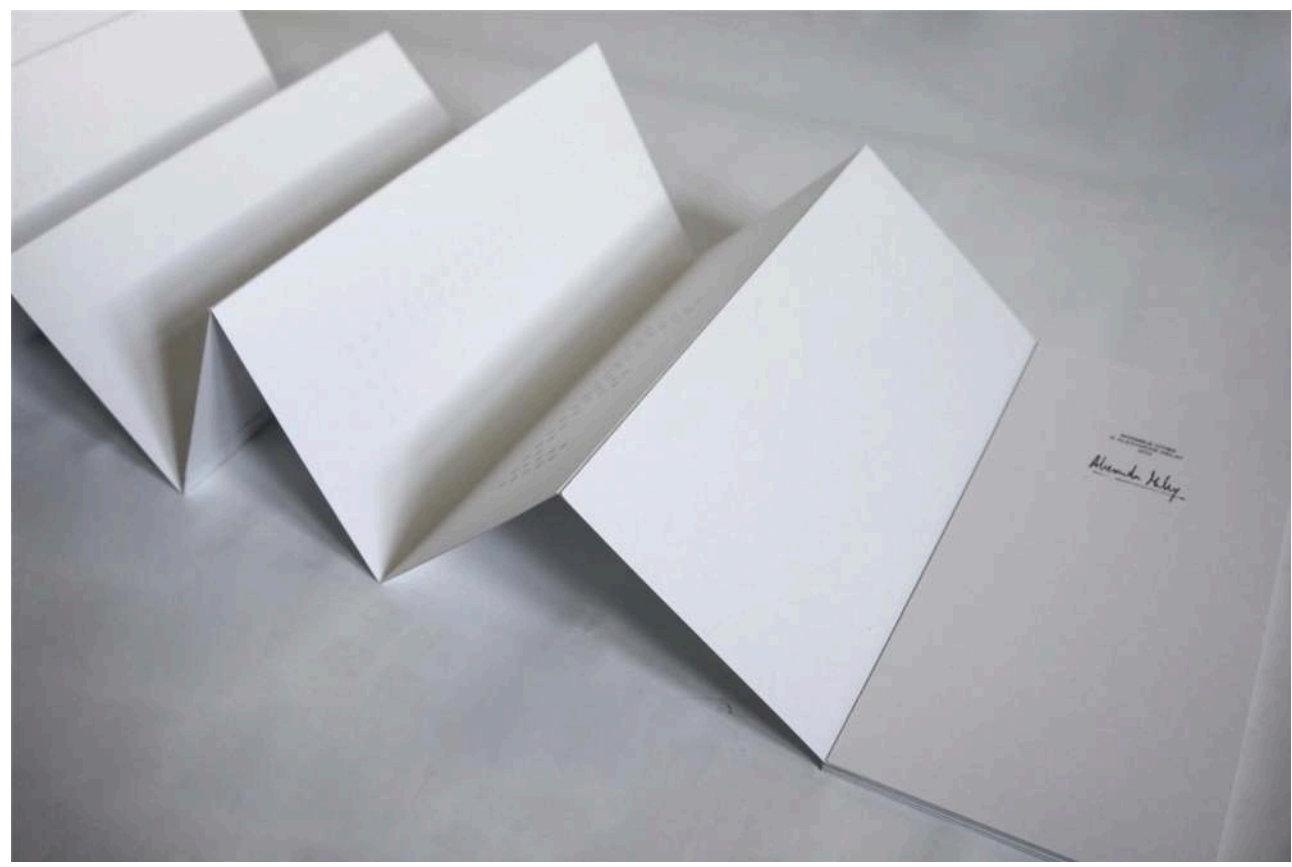

(c) Alexandre Melay. 
Figure 8. Transparence, feuilles transparentes et retranscription gravée du texte bouddhiste shakyo, le Hannya-Shingyo (Sûtra du Cœur), sous coffret plexiglas, $160 \times 220 \mathrm{~cm}, 2015$.

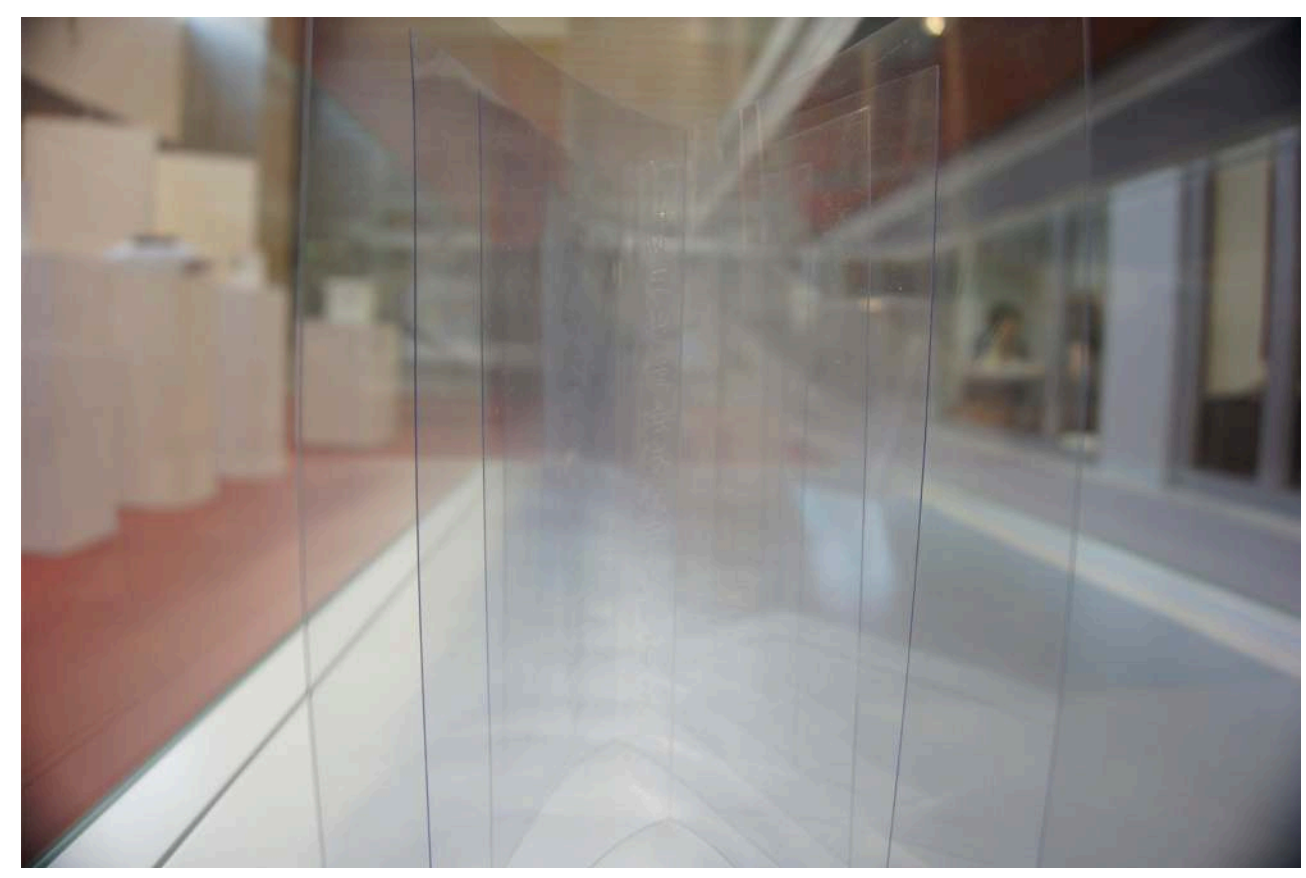

(C) Alexandre Melay.

vм : Dans les œuvres que l'on a évoquées, le livre s'articule grâce à des pliures ou se déroule en maintenant un imaginaire narratif. En revanche dans d'autres de vos œuvres, comme les livres-maquettes, vous proposez quelque chose de très interactif puisque les feuillets se trouvent dans une boite ou sont enveloppés, mais ne sont ni reliés ni attachés. Cela dirige-t-il le lecteur vers une liberté totale?

AM : Il est vrai que d'autres réalisations sont plus interactives, et génèrent une nouvelle relation spatiale puisque le corps se trouve davantage sollicité. Je trouve intéressant que le déplacement du corps et du regard puisse se faire au moyen d'interventions sur les percepts phénoménologiques de la structure constitutive de l'objet. Je pense que l'objet-livre prend sens lorsqu'il est pratiqué, lorsque le lecteur déplace les différents éléments. Le geste de feuilleter devient celui de détacher, défaire un nœud, démonter, remonter, ré-attacher, réagencer, ou refermer... Que ce soit des affiches, des intercalaires, des filtres, ou des feuillets libres, tous ces éléments et objets non reliés permettent une combinaison variée de lectures et de découvertes. C'est le cas des "livres-maquettes", des objets illustratifs, mais aussi explicatifs. Ces objets-livres peuvent ainsi s'apparenter à des archétypes, des matrices, des systèmes de représentation; à des objets se situant entre l'abstraction de la représentation architecturale et la forme physique d'un bâtiment. C'est le cas du "livre-maquette" Fragments (2013) qui se présente sous la forme d'une boîte et qui fait référence à la surface de la chambre de thé japonaise, un espace à la superficie réduite (Fig. 9). J'ai choisi de réaliser cet objet-livre pour qu'il puisse se déplier dans l'espace, à l'aide de plusieurs éléments autonomes non reliés et disposés par strates dans la boîte. Ici, la manipulation favorise le déploiement du livre suivant le modèle plié des maisons de thé, un style de maquette en papier japonais okoshi-ezu. Il communique l'information à travers un modèle qui se monte selon un système de pop-up et permet l'ajout d'inserts et d'onglets, de crochets et de notes si bien que les matériaux, les dimensions et les textures peuvent être répertoriés. Ainsi, j'ai voulu 
que chaque page soit comme un mur, une cloison de la chambre du thé ; j'ai traité chaque élément différemment avec des papiers et des matières spécifiques rappelant les textures utilisées dans la construction de cet édifice, fait de matériaux industriels et naturels, de bois, de bambou ou d'écorces d'arbres.

Figure 9. Fragments, multiples éléments dans boîte en carton : feuillets libres, gravures, photographies, $260 \times 335 \mathrm{~mm}, 2013$.
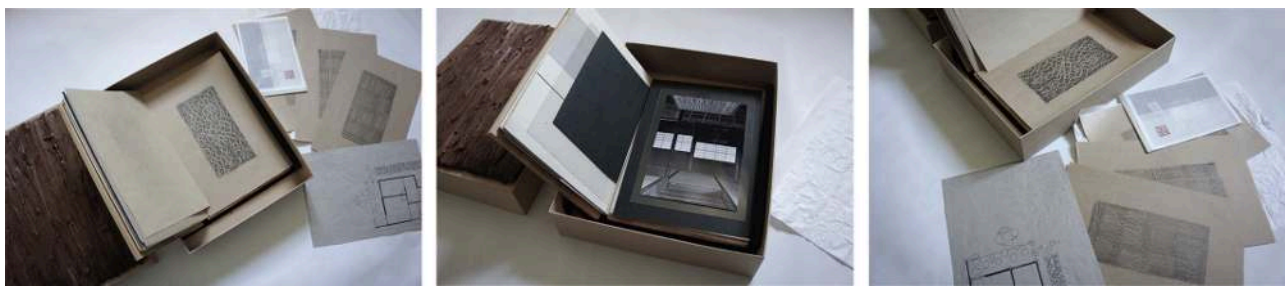

(c) Alexandre Melay.

Dans 形 KATACHI (2014), c'est la forme architecturale des maisons de ville japonaises qui m'a inspiré (Fig. 10). J'ai conçu cet objet-livre comme une forme qui sort d'un moule, un prototype au format carré. Les différents éléments au format carré sont une référence au style japonais. De même, la couverture et les différentes pages représentent les couches translucides de la maison japonaise, faite de vides, de blancs, d'intervalles. Les éléments que j'ai utilisés et les diverses feuilles sont comme des matériaux architecturaux interchangeables, en référence aux parois coulissantes faites de papier translucide que l'on déplace dans la maison japonaise. Les découpes formées sont autant de trames, de fragments, de textures qui traduisent ces éléments architecturaux.

Figure 10. 形KATACHI, multiples éléments découpés dans boîte en bois, 160 x 160 mm, 2014.
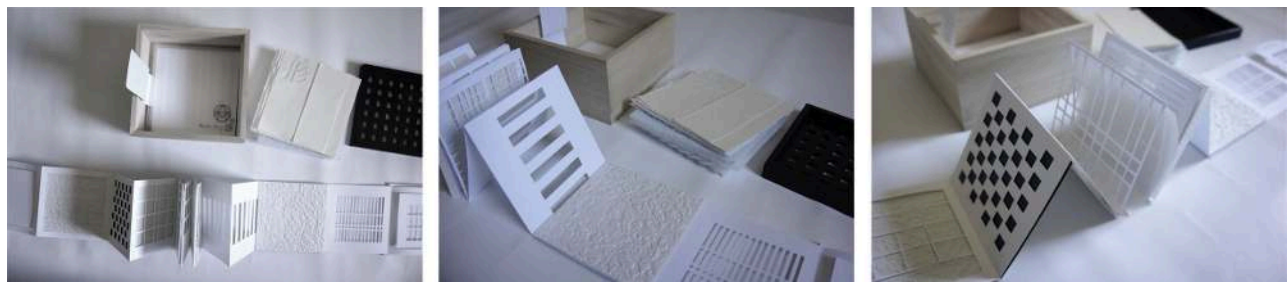

(c) Alexandre Melay.

Figure 11. Timeless, divers éléments, photographie, texte dans coffret noir, 290 × 290 mm, 2014 .
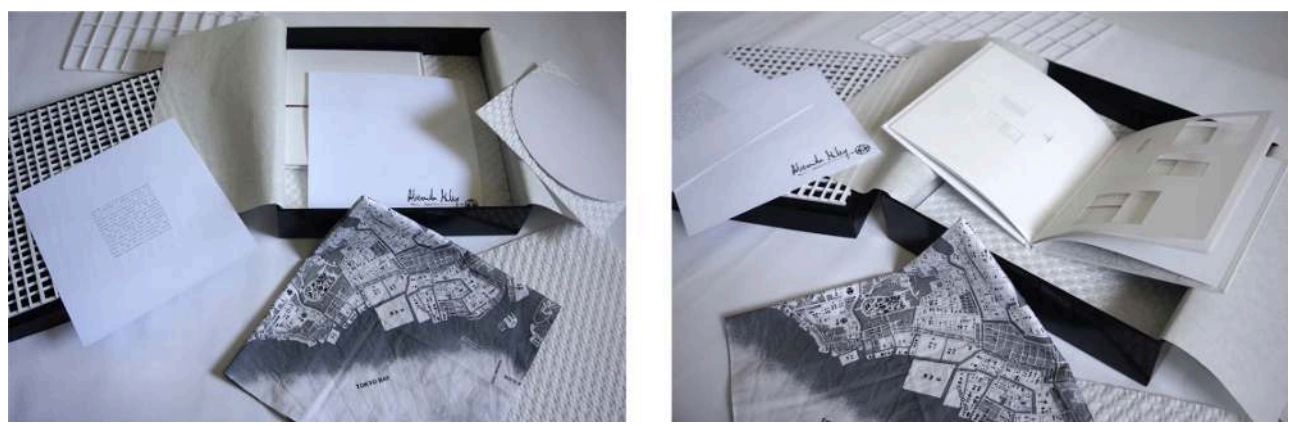

(c) Alexandre Melay. 
Grâce au même procédé, j'ai réalisé Timeless (2014), un livre au système modulable avec des éléments formés à partir de la boîte carrée, à l'image d'une matrice (Fig. 11). À l'intérieur, les différents éléments sont eux aussi au format carré. Le livre blanc à la forme carrée et à la couverture rigide fermée représente l'espace de l'architecture et reflète aussi l'extérieur de la maison par son enveloppe. Chaque page comporte des vides qui ont été soustraits à un mur de la maison. Il y a des lignes, des découpes, un style épuré et minimaliste qui se réfèrent à l'architecture contemporaine japonaise. J'ai ajouté un texte imprimé au format carré que l'on découvre à l'ouverture de la boîte. Le vide des structures de l'architecture japonaise se reflète dans les trois ossatures vides de l'objet, en référence aux squelettes de bois de l'architecture traditionnelle. De par leur format et leur forme, ces trois livres sont à comprendre comme des maquettes d'architecture qui se déplient dans l'espace, hors du support du livre.

vм: Comme vous venez de l'expliquer, plusieurs de vos œuvres se transforment en architecture et proposent une modélisation expérimentale de l'espace. Elles invitent à articuler les pleins et les vides et à recréer un espace hors de celui de la boîte. Ces boîtes sont un espace à déployer. C'est un peu comme si l'utilisateur - on ne peut plus parler ici de lecteur - devenait sculpteur, architecte, cartographe puisqu'il déplie l'œuvre à son gré, éparpille des éléments, conquiert un espace nouveau. Qu'advient-il alors de la question du format?

AM : D'autres créations sont encore plus proches de la forme d'une sculpture, d'un véritable objet en trois dimensions qui donne une nouvelle spatialité au livre. Ces livres, je les appelle des "livres-édifice", à l'image d'une architecture. L'objet-livre intitulé Mineral (2014) a été réalisé à partir de pages pliées, et qui une fois posées à la verticale forment un bâtiment (Fig. 12). J'ai conçu huit planches pliées qui demandent à être placées comme des façades d'édifices interchangeables. La couverture, en trois dimensions, faite de cristaux en papier plié, rappelle les architectures contemporaines débridées présentes dans l'espace urbain japonais. C'est aussi les architectures atypiques comme la Ring House qui ont inspiré le "livreédifice" Process (2014) (Fig. 13). J'ai souhaité réinterpréter dans une mise en livre sculpturale, la structure de l'édifice dans une alternance de vides et de pleins, de transparences et de dissimulations. L'architecture fait partie du livre, grâce aux découpes que j'ai effectuées directement dans les pages de l'ouvrage. À l'ouverture, le livre originel se transforme en une sculpture. Un procédé que j'utilise aussi dans l'objet-livre Invisible (2013) dans lequel c'est une architecture transparente qui se déploie à l'ouverture d'un ancien livre japonais (Fig. 14).

Figure 12. Mineral, papiers et dessins au graphite, non relié, 300 × 260 mm, 2014.
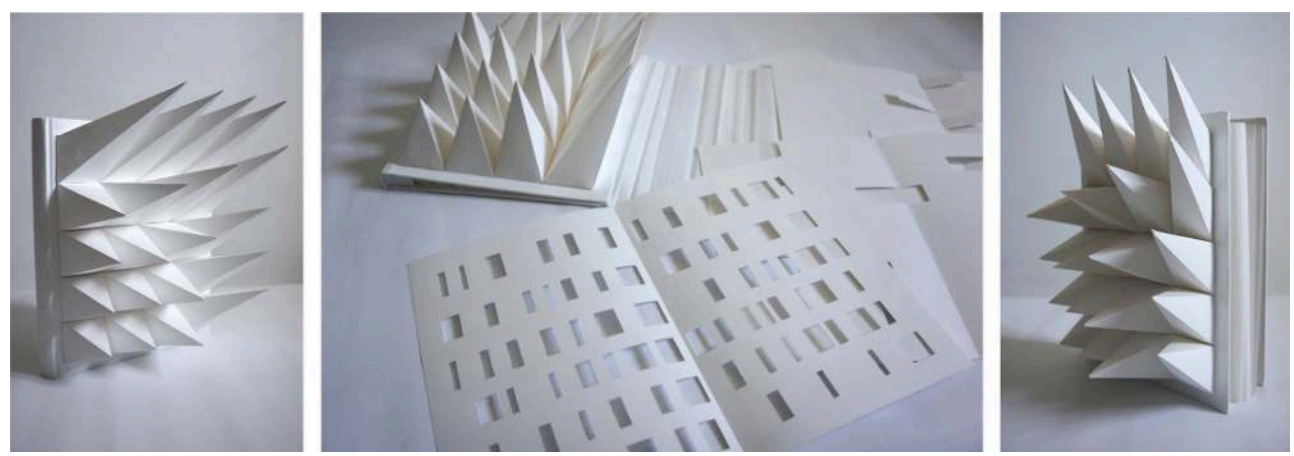

(c) Alexandre Melay. 
Figure 13. Process, livre découpé, peinture acrylique et image photographique, 160 × 245 mm, 2014 .

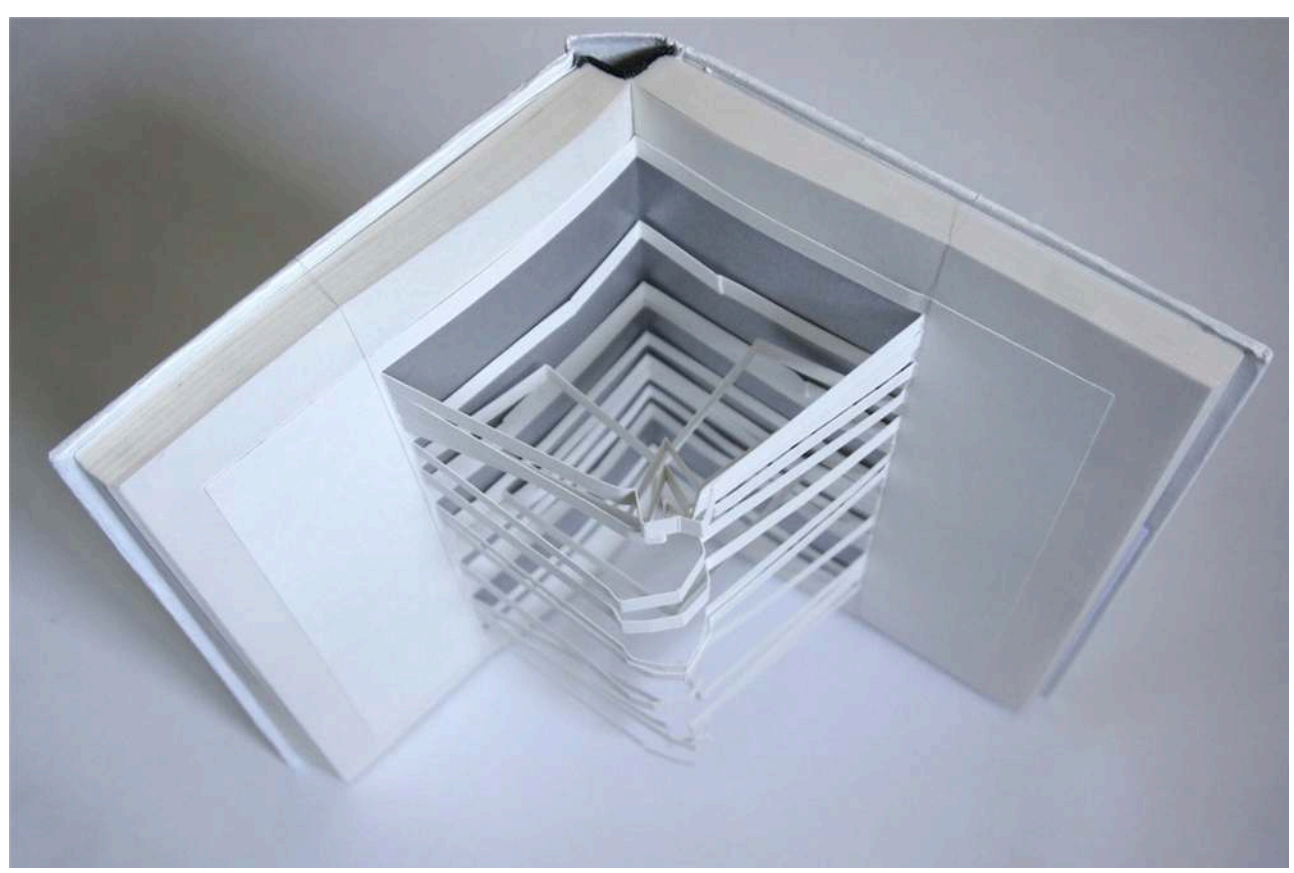

(c) Alexandre Melay.

Figure 14. Invisible, livre japonais et matière plastique, 300 x 210 mm, 2013.
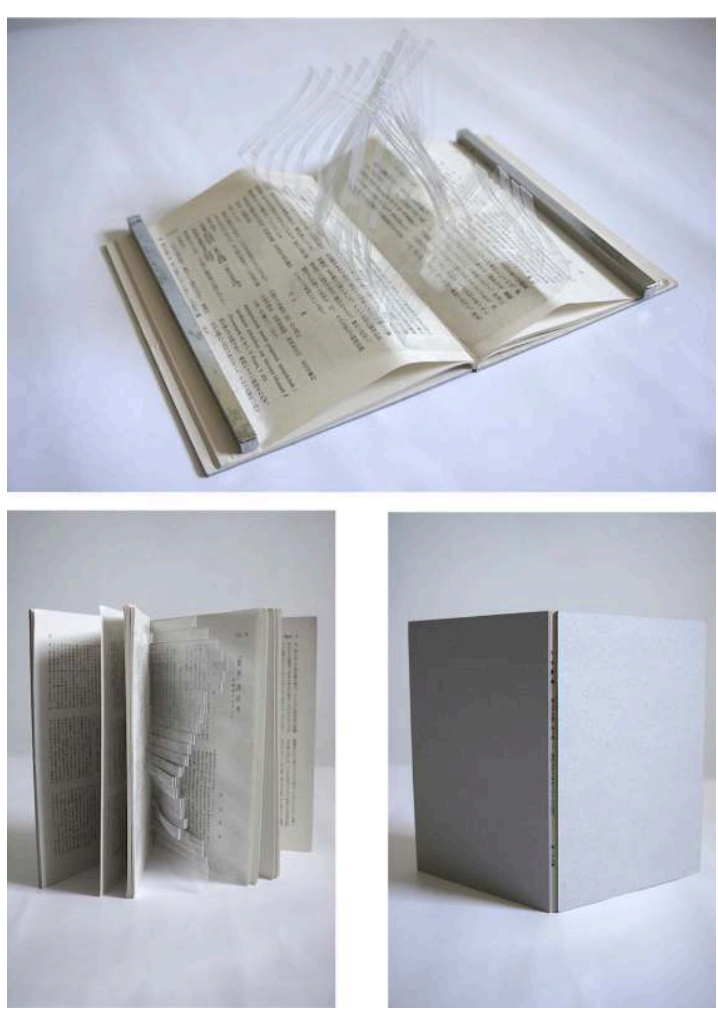

(c) Alexandre Melay. 
VM : Ces objets sont-ils encore des livres?

AM : C'est vrai, certaines conceptions formelles invitent à une autre expérience de lecture. Le livre devenant un élément dans la réalisation de l'objet et non plus le médium. Dans le cas de l'objet-livre Silence [R] (2013), c'est l'architecture du jardin sec du Ryoan-ji à Kyoto et toute la conception bouddhique zen du vide qui m'ont inspiré (Fig. 15). Dans son titre j'ai aussi voulu rendre hommage à l'œuvre de John Cage, 4'33" (1952), un morceau souvent décrit comme « quatre minutes trente-trois secondes de silence ». La forme de cette création est résolument atypique puisqu'elle se compose d'un livre noir tatoué de la lettre $\mathrm{R}$ pour le $\mathrm{R}$ de Ryoan-ji ; j'ai choisi de poser le livre sur un coussin blanc de format rectangulaire, un élément matériel très présent dans la médiation zen. Pour évoquer l'espace du jardin, j'ai ensuite creusé et évidé le livre, je l'ai rempli à nouveau avec des petites formes géométriques qui représentent les quinze pierres qui composent ce célèbre jardin japonais. Dans ce type de réalisation, on se situe davantage dans l'idée d'un objet que d'un livre à proprement parler, tout comme dans Fade out, fade up (2016) (Fig. 16). Un objet qui s'inspire aussi du jardin japonais fait de sable blanc, que j'ai souhaité visuellement silencieux, mais qui communique une idée par sa seule présence physique. Formé de deux boîtes en plexiglas superposées qui contiennent, dans l'une, de simples feuillets pliés et imprimés de plusieurs phrases interchangeables qui font référence à la notion japonaise de la vacuité ; et dans l'autre, du sable blanc qui demande à être secoué, pour laisser se dévoiler une forme vide, celle du cercle de la concentration mentale. Dans le cas de cet objet, volontairement dénué de signes visuels ou linguistiques, c'est la seule présence du contour formant un cercle vide ainsi que le titre qui définit le champ conceptuel et relativement mystérieux de l'objet-livre.

Figure 15. Silence [R], livre découpé 210 x 140 mm sur cousin de tissu blanc, 500 × 300 mm, 2013.
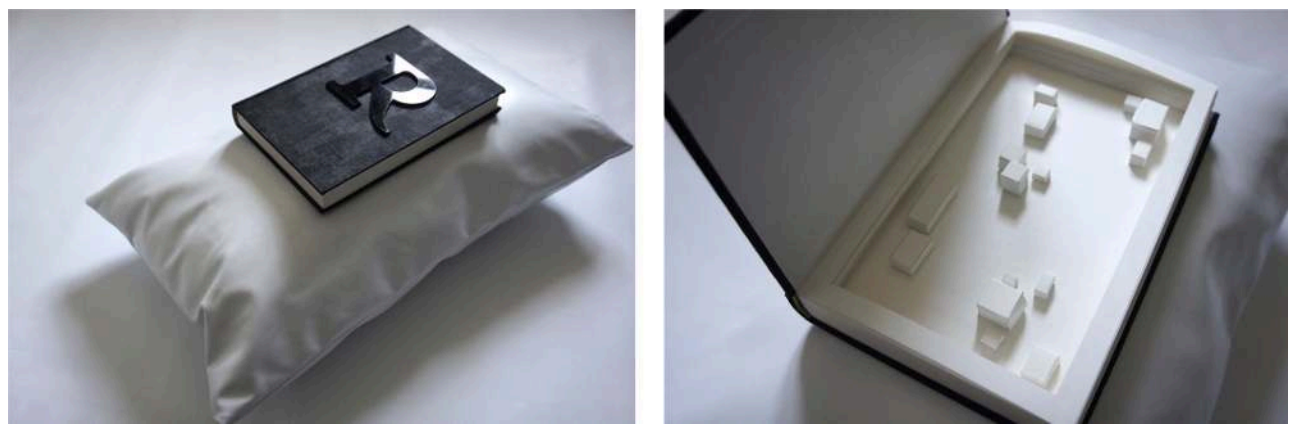

(c) Alexandre Melay.

Figure 16. Fade out, fade up, textes sur papier glacé, photographie, boîtes en plexiglas, sable blanc, $160 \times 220$ mm, 2016 .
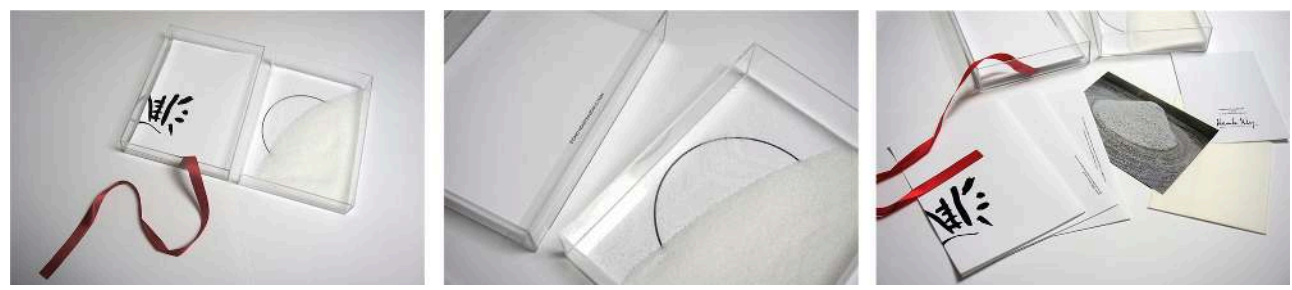

(C) Alexandre Melay. 
Dans Urbanized (2014), le corps du livre permet de recréer l'espace architectural et urbain à travers les pleins et les vides des pages intérieures qui ont été évidées (Fig. 17). Ce sont les vides des découpes qui illustrent l'espace urbain de la ville japonaise, mais aussi des façades de constructions où seules les enseignes aux néons verticaux sont représentées. Les découpes forment des zones, des entrailles, les entre-deux qui traduisent l'organisation de l'espace de la ville labyrinthique, les entrailles du livre comme les entrailles de la ville - l'urbanité, l'espace-intervalle en référence au concept du ma japonais. De même, j'ai choisi de conserver et de déposer les éléments typographiques découpés dans deux boîtes transparentes en plexiglas au format rectangulaire à l'image d'un cercueil. Ces restes de l'espace forment des strates et deviennent la mémoire des constructions détruites dans un jeu de puzzle infernal.

Figure 17. Urbanized, livre et éléments découpés dans boîtes en plexiglas, dimensions variables, 2014.
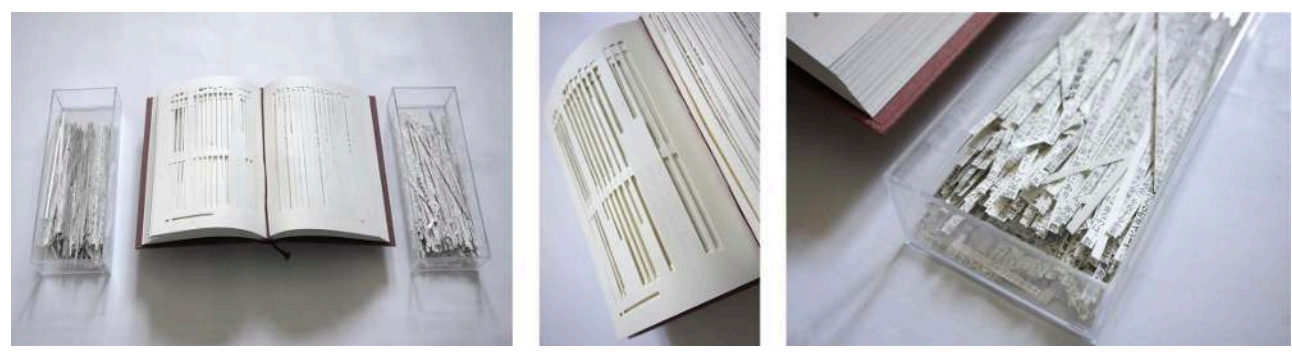

(c) Alexandre Melay.

On le voit à travers ces derniers objets, toute cette combinaison d'éléments incite à concevoir le livre comme un objet physique, en trois dimensions; des objets que l'on a envie de toucher, d'ouvrir, de saisir et de manipuler dans ses mains. La lecture impose le mouvement des yeux du lecteur qui manipule les différents éléments puisque la forme génère une nouvelle relation sensorielle avec l'objet-livre où tous les sens se trouvent sollicités. C'est le format du livre en tant que tel qui détermine le type de relation que nous allons avoir avec lui lors de sa lecture. Entre l'espace intime d'un petit format et l'espace plus public d'un grand format, c'est précisément cette relation de corps à corps ou de face à face qui caractérise et singularise chaque espace de lecture. La forme donne au livre d'artiste la possibilité de dépasser les contraintes d'une structure pour reconfigurer son identité en fonction d'un contenu dans une conception spatiale spécifique.

vм : II y a également, dans nombre de vos propositions, un jeu sur les textures qui suggère que votre approche n'est pas uniquement conceptuelle, mais intègre la matérialité du papier. Dans certaines œuvres, vous utilisez du papier calque par exemple. Comment forme et matière interagissent-elles?

AM : Ma pratique passe effectivement par un véritable travail sur les matériaux et les textures. Je construis un objet-livre en utilisant des matériaux, des outils, des matières, des papiers, différents supports, différentes couleurs, différentes textures, qui sont comme autant d'éléments d'architecture, qui structurent l'espace du livre. Chaque élément matériel est un élément constructif du livre comme ceux d'un bâtiment en architecture. Pour cela, je travaille beaucoup avec des techniques de juxtaposition, de stratification, mais aussi d'empilements, de superposition de différents éléments de bois, de plastique, de papier... Une notion de surfaces, surfaces des papiers, des textures, des matériaux que l'on retrouve dans le livre Traces (2014), 
un objet-livre composé d'une série de frottements que j'ai réalisé à partir des structures de l'architecture traditionnelle japonaise (Fig. 18). Le livre est réalisé par les techniques de frottage et de décalquage qui font ressortir tous les défauts de la matière exposée au passage du temps et donc à son histoire. De même, j'ai choisi d'enrouler le carnet dans une natte en fibre naturelle qui rappelle la matière vivante, son authenticité, son allure douce et tactile, son écorce vieillie et son aspect lustré produit par le temps, tout comme les différents frottements qui traduisent la force et la vigueur de la matière brute du bois, sa vitalité.

Figure 18. Traces, carnet de gravures, frottements au crayon noir ; enroulé dans une natte en bambou, dimensions variables, 2014.

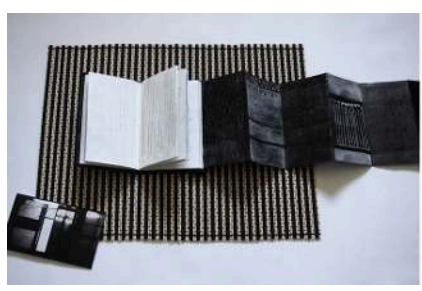

(c) Alexandre Melay.
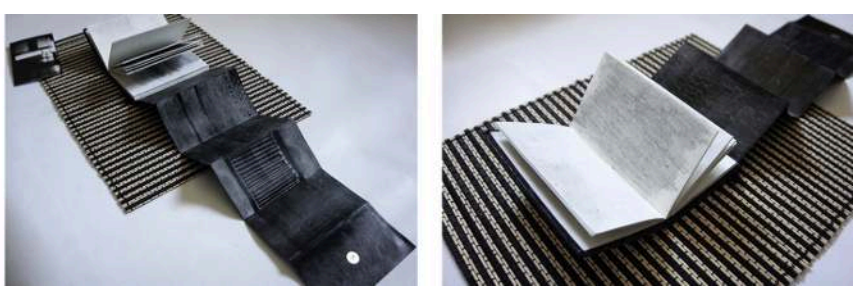

Toutes ces matières convoquent le concept d'haptique, "le toucher par les yeux", comme dans l'objet-livre Generative forms (2013) qui se présente sous la forme de plusieurs feuilles de papier japonais non reliées sur lesquelles des formes géométriques, des cercles, autant de motifs répétés à l'encre noire, déséquilibrés par des éléments en or et en argent se confrontent, s'entrelacent, s'entrechoquent et suggèrent de multiples histoires, de multiples découvertes (Fig. 19). C'est au lecteur de choisir de former lui-même le livre en déplaçant à sa guise les pages interchangeables.

Figure 19. Generative forms, encre de Chine et peinture acrylique sur papier japonais, non relié, 250 x $340 \mathrm{~mm}, 2013$.
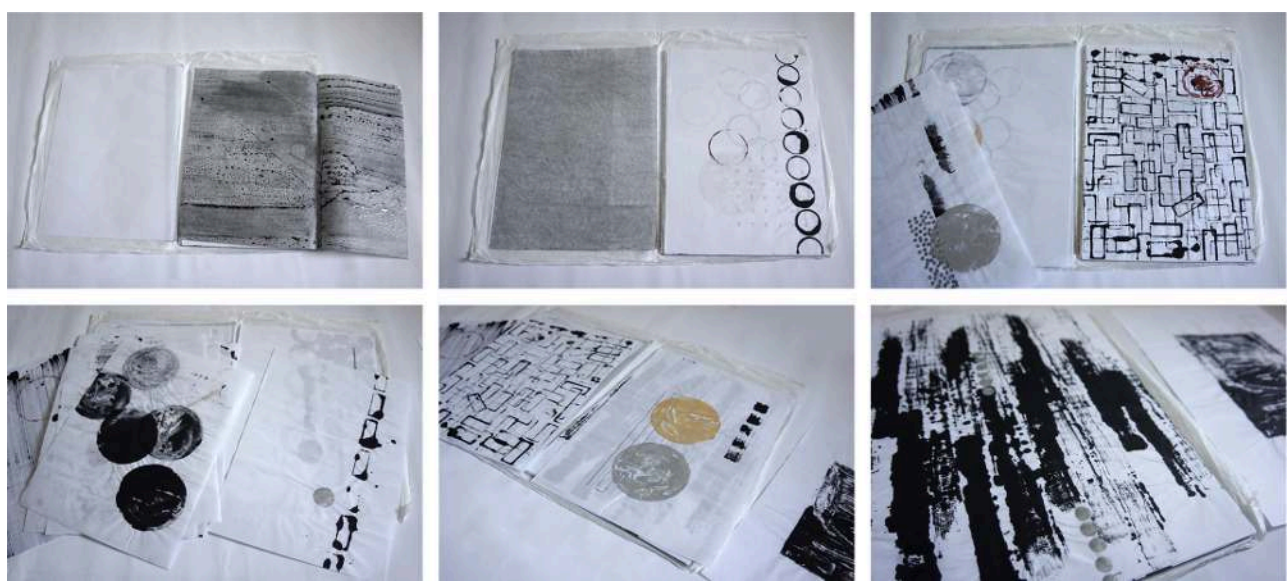

(c) Alexandre Melay.

Dans Anthropomorphism (2014), l'objet est aussi constitué d'une multitude de surfaces de papier calque translucide à l'aspect lisse et soyeux qui invite au toucher (Fig. 20). Le choix du format du livre s'est porté sur une forme verticale légèrement allongée, utilisée notamment dans la calligraphie japonaise et plus précisément dans la 
réalisation de kakemono, ces peintures ou calligraphies japonaises installées dans un rouleau pour y être suspendues verticalement. Ce format correspond à la spatialité que requiert la calligraphie, réalisée généralement de haut en bas sur des supports verticaux étroits, mais s'étirant en longueur. Les différentes feuilles libres regroupées et serrées entre deux plaques de plexiglas, comme mises sous presse, décrivent l'idéogramme de l'arbre en prenant une forme quasi vivante au fil des pages en se superposant, ce qui crée une sorte de mouvement de strates dans un véritable jeu d'ombres et de transparences.

Figure 20. Anthropomorphism, peinture acrylique sur papier calque, 20 feuillets libres non reliés, couverture en plexiglas, 450 × $210 \mathrm{~mm}, 2014$.
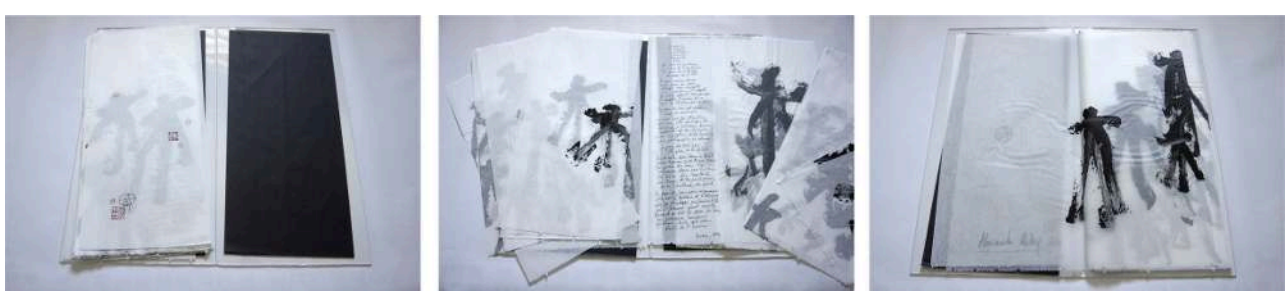

(c) Alexandre Melay.

C'est avec le même système de feuillets libres que j'ai composé l'objet-livre Évanescence (2012) à partir de plusieurs pages pliées et présentées à la manière d'un portfolio (Fig. 21). La couverture en plastique perforée d'une multitude de petits trous invite au toucher et à l'ouverture du livre. Là encore, l'importance est donnée à la matière : à l'intérieur, j'ai choisi d'utiliser des feuilles simples aux surfaces variées qui rappellent les surfaces de l'architecture contemporaine, et rappelle l'expérience visuelle de la ville de Tokyo faite de signes, de graphismes et de surfaces. Chaque planche est interchangeable, alternant entre des graphies monochromes imprimées et des feuilles semi-transparentes de différentes teintes qui viennent éclabousser le flux monochrome comme pour rappeler qu'à la nuit tombée, la ville se vêt d'une multitude de couleurs vives. Comme des signes mouvants défilant sur des surfaces de verre transparentes qui ressemblent à des écrans, le livre prend forme à travers différentes surfaces de papiers, de textures, de matériaux ainsi qu'avec la présence de la photographie. La découverte du livre passe par la manipulation des différents éléments, les feuillets sur lesquels des filtres peuvent être glissés, intercalés sans restriction, permettant ainsi de pénétrer dans une ville textuelle.

Figure 21. Évanescence, feuillets avec textes et photographies, impression numérique, $250 \times 300$ $\mathrm{mm}, 2012$.
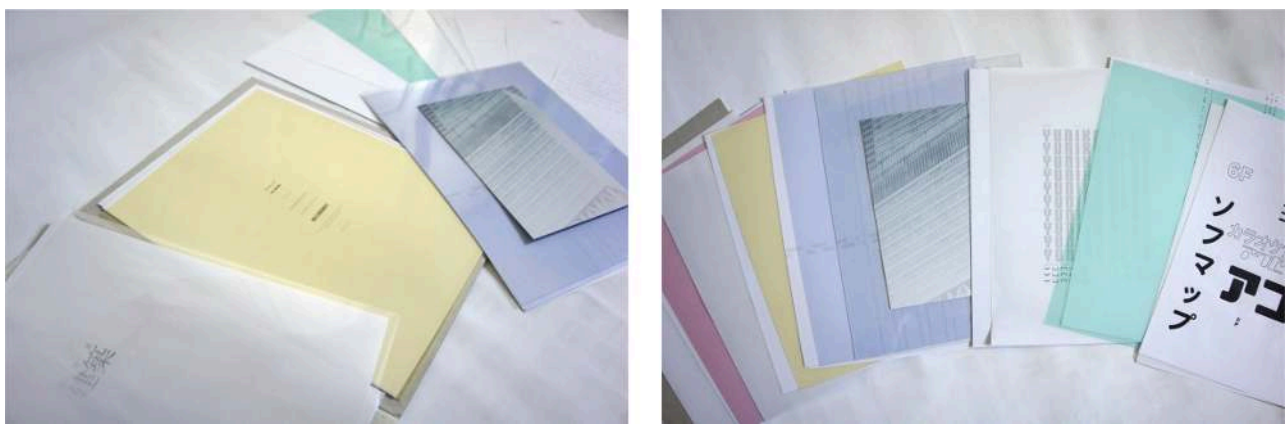

(C) Alexandre Melay. 
Dans l'objet poétique Snow, silence, and time, (2015), la matérialité du papier est davantage mise en avant, puisqu'elle traduit l'expérience du froid et de la neige de l'hiver ; le papier japonais est associé à la forme de l'objet tout en couches de papier et de tissu (Fig. 22). Cet objet fait de matières, de textures, de surfaces, demande à être manipulé, touché, ouvert. L'ensemble est enroulé dans une matière blanche qui rappelle une couche de neige épaisse. Le livre relié à la japonaise est réalisé avec du papier japonais particulièrement épais, absorbant et aussi moelleux que la neige. Sur les différentes pages virevoltent des flocons tracés très légèrement au crayon. Elles sont entrecoupées par des inserts sur lesquels des poèmes japonais haikus sont écrits à la main. J'ai choisi, pour réaliser la couverture du livre, un morceau de tissu d'un vieux kimono indigo qui maintient le livre à l'abri du froid comme un vêtement qui protège et réchauffe le livre enneigé. Le livre est accompagné d'une planche de bois creusée à la main dans laquelle est placée une photographie enveloppée dans un papier calque glacé et froid: la photographie d'un bambou blanc, elle-même placée dans une enveloppe de papier calque translucide, évoque une plaque de givre.

Figure 22. Snow, silence, and time, multiples éléments ; livre de dessins sur papier japonais, reliure japonaise, poèmes et photographies ; enroulé dans un tissu blanc, dimensions variables, 2015.
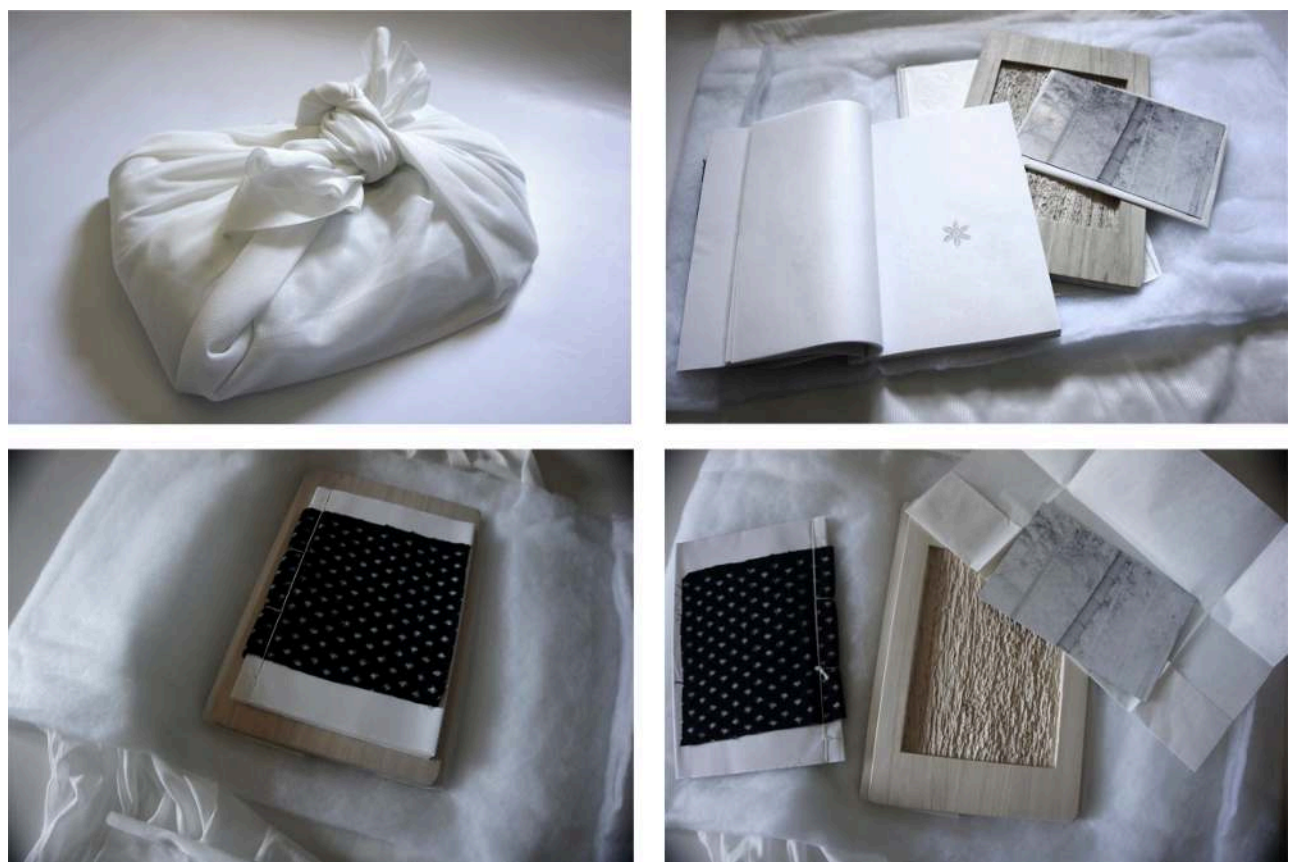

(c) Alexandre Melay.

vм : Ce que l'on voit dans ces divers déploiements matériels et ces dispositifs haptiques, c'est que le livre, associé dans nos cultures à un savoir intellectuel et abstrait, c'est-à-dire au signe, devient un objet sensible où corps et esprit, matériau et image sont réconciliés. La connaissance, contenue dans le livre-cadre n'est plus liée au pouvoir ni à la maîtrise du monde. Vos livres sont des espaces sensibles où un dialogue se déploie entre l'homme et le monde. Ils sont une rencontre. Tout autre chose en effet qu'un codex ou une encyclopédie! S'il n'y a plus de récit, de précepte ou de définition, il y a néanmoins l'évocation d'une expérience, l'émergence de souvenirs grâce aux matières. Au premier abord, vos objets de taille réduite convoquent un imaginaire de l'espace intime que Gaston Bachelard associe au coffre ou au tiroir. Pourtant, à l'intérieur, vous placez des cartes, des 
plans, des photographies qui ouvrent un espace plus grand. Deux spatialités se rencontrent; il y a des jeux d'échelles qui croisent les jeux de format.

AM : Effectivement, ce sont les cartes postales, les cartes géographiques ou encore les photographies, d'autres formes d'imprimés qui permettent d'agrandir l'espace du livre. Comme ces éléments naturels, de petites feuilles d'érable séchées que j'ai rassemblées dans un coffret en bois pour l'œuvre Kyôto-Mujô (2013) (Fig. 23). J'ai choisi le titre Mujô qui renvoie au temps japonais de l'éphémère et qui caractérise aussi les photographies, traitées en noir et blanc, comme si elles traduisaient une certaine nostalgie, ce sentiment japonais du passage du temps, comme celui des feuilles qui changent de couleurs. Par sa forme multiple, avec ses différentes enveloppes, j'ai pensé cet objet-livre comme une boîte de souvenirs, avec l'idée d'un livre émotionnel traduisant un phénomène naturel, celui de la "chasse aux couleurs" des feuilles d'érable à l'automne au Japon, qui sont ici conservées dans des enveloppes transparentes laissant apparaître leur changement de couleur, passant du vert, au rouge puis à l'orangé; un moment transitoire très caractéristique de l'esthétique japonaise et qui rappelle que la vie est éphémère. J'ai aussi souhaité que le lecteur puisse se retrouver dans une position de décideur, où il peut avec tous ces éléments déployer lui aussi sa propre histoire, son propre imaginaire.

Figure 23. Kyôto-Mujô, éléments multiples : photographies en noir \& blanc, éléments naturels, boîte en bois, $180 \times 190 \mathrm{~mm}, 2013$.

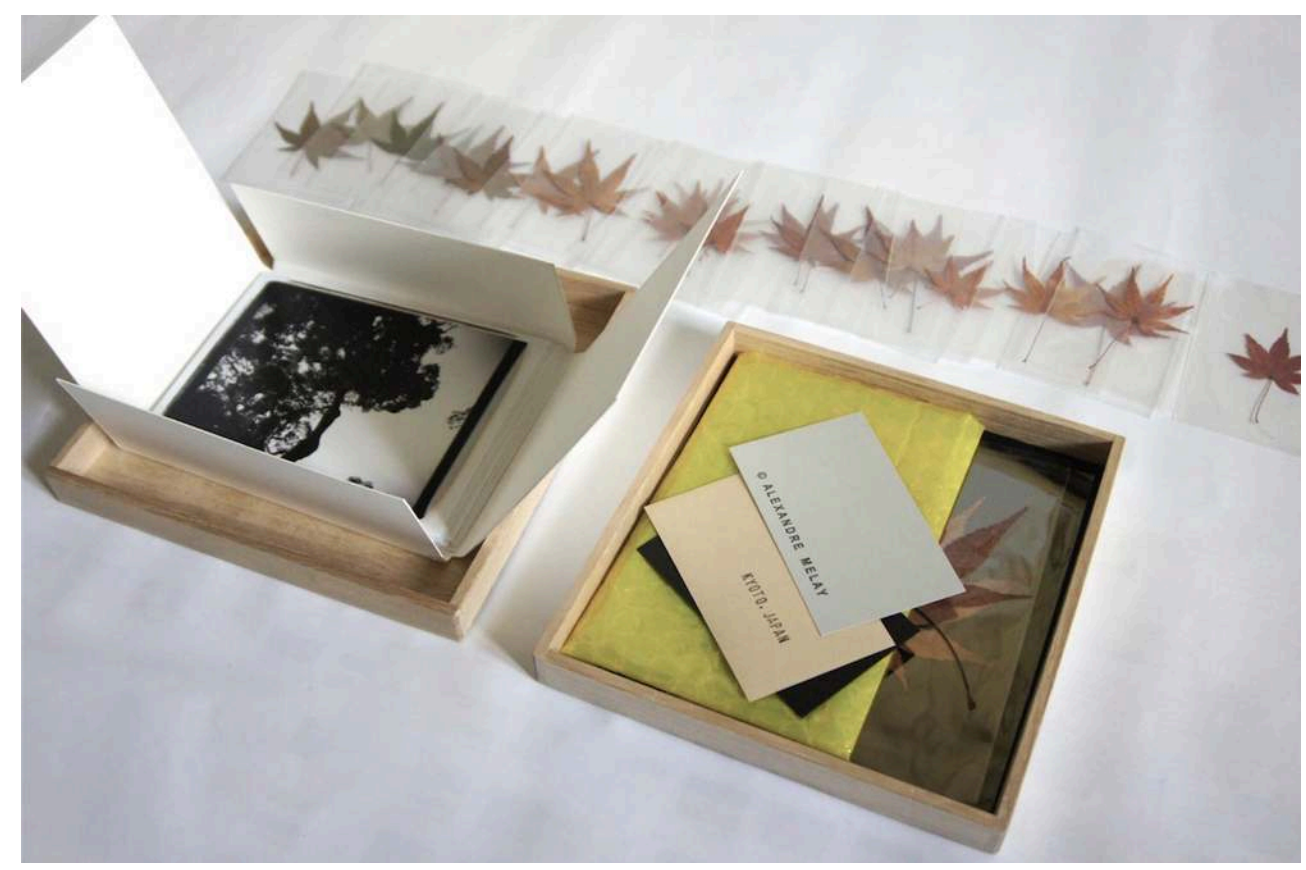

(c) Alexandre Melay. 
Figure 24. In-between, textes et photographies en couleur, découpages, couverture rigide, $190 \times 190$ $\mathrm{mm}, 2014$.

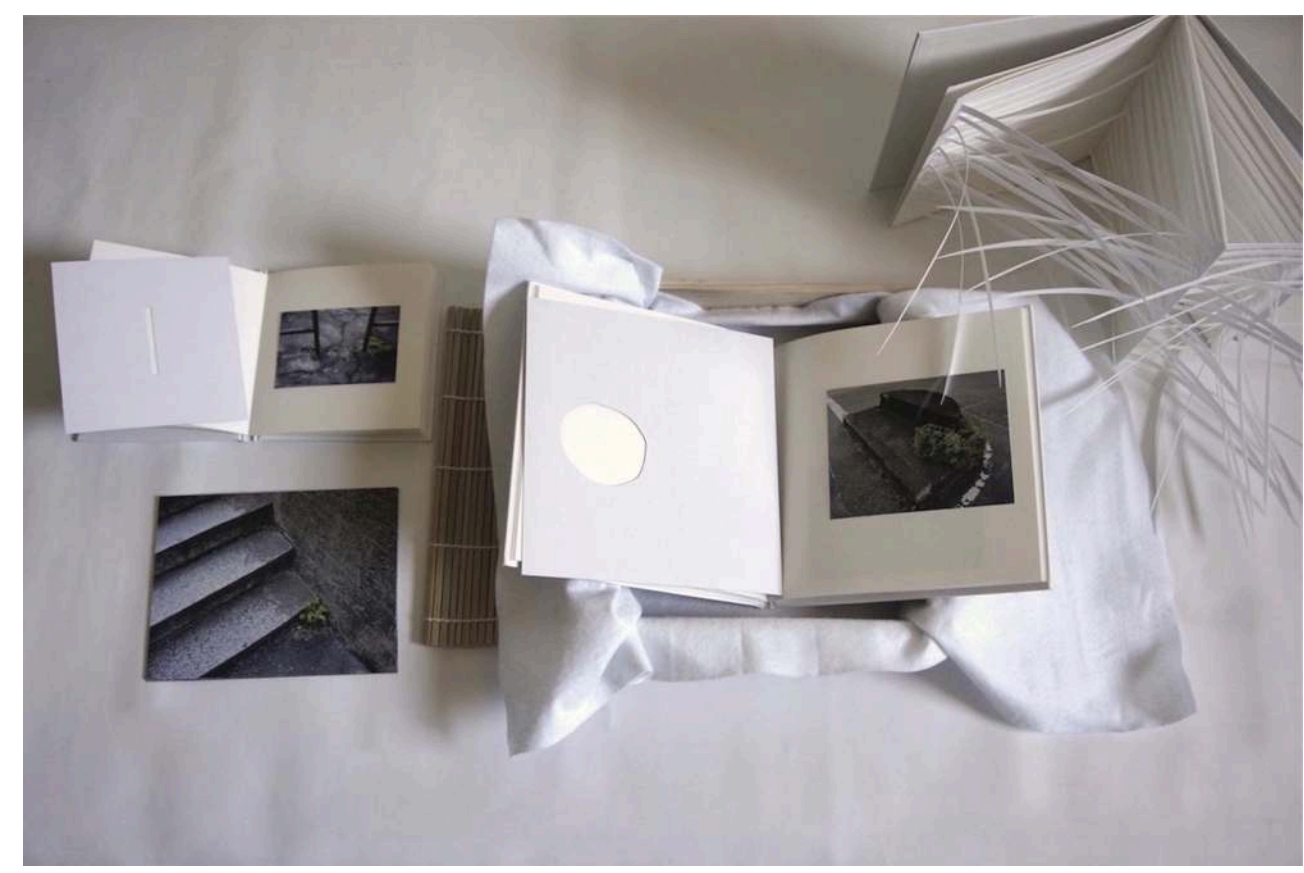

(c) Alexandre Melay.

Ces photographies prises dans la ville de Tokyo aux prémices du printemps composent l'objet-livre In-between (2014) (Fig. 24). Ici aussi, la forme même de l'objet est complexe puisque l'œuvre est constituée de trois livres au format carré et à la couverture blanche. J'ai choisi d'envelopper ces trois livres dans de la feutrine blanche, et placé l'ensemble dans une boîte en bois clair fermée sur le dessus par une natte de bambou. Cet objet-livre contient une variété de supports enveloppants, écrans ou masques. Ils valent pour ce qu'ils cachent ou protègent. Mais une fois déployé, le livre prend une autre dimension. À l'intérieur le livre prend du volume, de l'espace et, sur les images, des herbes nouvelles ainsi qu'une nature qui résiste à l'urbanité se dévoilent. J'ai réalisé ces photographies de mauvaises herbes parce qu'elles symbolisent la force et l'énergie de la nature et semblent échapper à tout contrôle en prenant racine entre les fissures. De même, j'ai voulu traduire ces images dans le livre en découpant les pages en fines tiges. Elles sont un peu parasites, comme de mauvaises herbes poussant dans les aspérités des murs, et elles induisent une échappée verte et poétique hors des pages blanches du livre.

Dans IMA, I am here (2015), le livre se déploie aussi grâce à la photographie (Fig. 25). La forme est façonnée par son contenu, par l'accumulation de plus d'une centaine de photographies empilées et présentées dans une boîte en plexiglas. Un carnet rouge permet de fermer la boîte. La déambulation, la circulation comme geste artistique se retrouvent dans la manipulation des photographies, de la carte, du mouvement des yeux et du corps. Le marquage en rouge sur une carte atteste de la présence physique. Les différents éléments procèdent tous du même format permettant ainsi d'être regroupés au sein de la même boîte, mais ils finissent, en se déployant, par devenir une carte. La carte, c'est aussi, l'élément qui forme le livre 2A (scale) (2013) (Fig. 26). Dans ce cas, c'est l'inverse qui est produit, la carte géographique japonaise ancienne ne se déplie pas, mais forme le livre après avoir été découpée en feuillets de 
taille égale puis reliées entre eux. Le livre demande à être manipulé et ouvert de manière à former un cercle de 360 degrés. Ce "livre-carte" avec ses différents fragments interroge la géographie et la notion même d'espace, puisque chaque morceau de carte est mélangé aléatoirement. Libre au lecteur de s'approprier un morceau de l'espace géographique.

Figure 25. IMA, I AM HERE, carnet d'écrits, photographies et carte postale, dimensions variables, 2015.

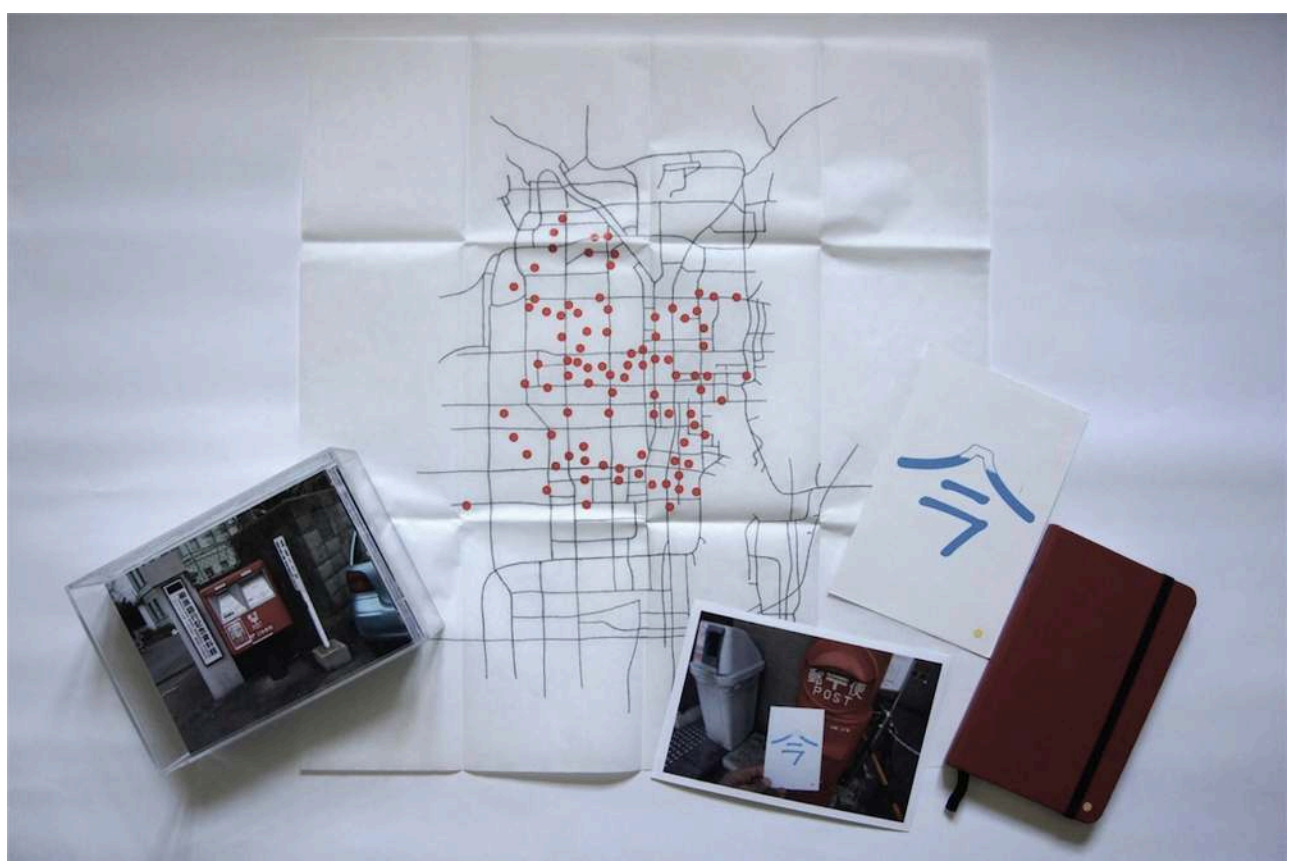

(c) Alexandre Melay.

Figure 26. 2A (scale), carte géographique fragmentée, dimensions variables, 2013.

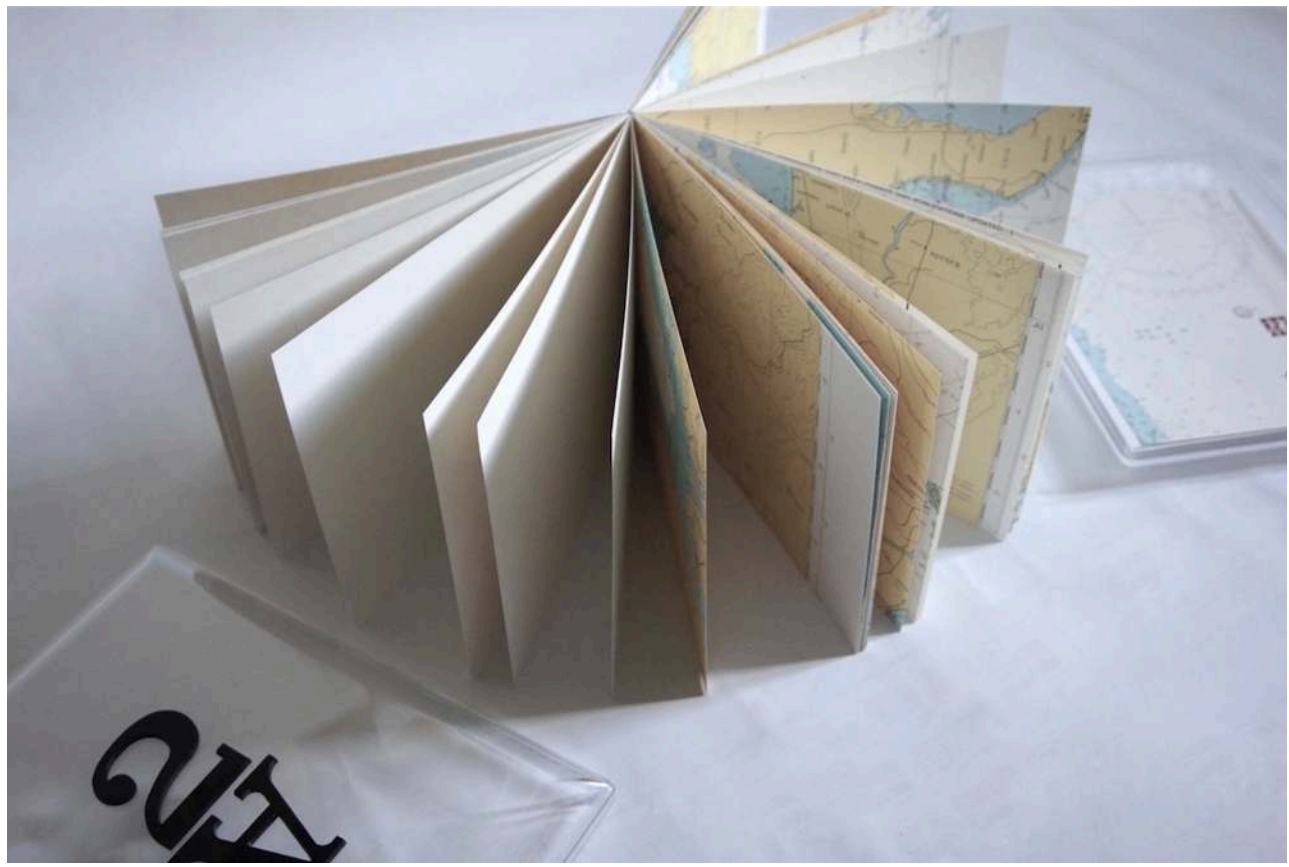

(c) Alexandre Melay. 
La notion d'enveloppement, avec la succession d'enveloppes, d'emballages (tissus, papier), d'emboîtements, renforce aussi la notion de dédoublement de spatialités, comme dans le "livre-boîte" intitulé [TIME] Strates (2013) qui demande à être ouvert, puis manipulé hors de sa boîte, car tous les éléments en papier sont pliés ; des pliages qui demandent toujours à être dépliés afin de dévoiler dans son ensemble l'impression de l'encre sur les différentes feuilles de papier, permettant ainsi une véritable démultiplication visuelle de l'œuvre (Fig. 27).

Figure 27. [TIME] Strates, encre de Chine sur papier japonais plié en plusieurs éléments, présentés dans une boîte en bois, 105 x 190 mm, 2013.
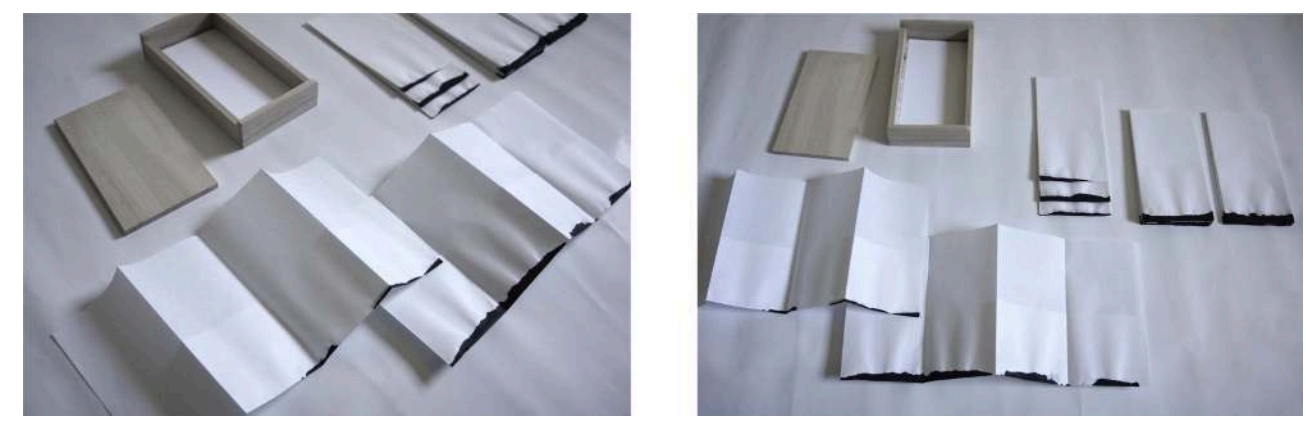

(c) Alexandre Melay.

VM : Vos déclinaisons du livre n'intègrent que très peu de texte. Pourquoi cette absence de l'écrit et des mots?

AM : Mes livres fonctionnent comme des textes et n'ont pourtant pas besoin d'être traduits par des mots. Mais leur sens n'est pas arrêté. Mes objets-livres sont néanmoins très souvent accompagnés de simples mots, de phrases, de citations, d'extraits d'ouvrages, ou de poèmes - et particulièrement de haiku, mais aussi de chiffres, ou de sons comme dans le "livre sonore". Intitulé System (2011), ce livre est composé de plusieurs feuillets de même format sur lesquels sont imprimées des photographies et des formes graphiques (Fig. 28). Ce sont dix images fixes extraites d'une vidéo en travelling réalisée à bord du train à grande vitesse japonais, présentées sur chaque double page, à droite, avec en regard, un nombre en couleur rouge qui correspond à la vitesse du train. La forme même du livre avec un rendu éclaté, déformé, se rapporte à la vitesse. Mais le plus important, c'est l'insertion d'une page-disque comprenant l'enregistrement sonore qui restitue le bruit mécanique du train. En confrontant les images à la bande sonore, je voulais que le lecteur puisse faire une expérience presque cinématographique, au fil des pages et au fil du son. 
Figure 28. System, feuillets imprimés, CD audio et boîte en carton gris, dimensions variables, 2011.

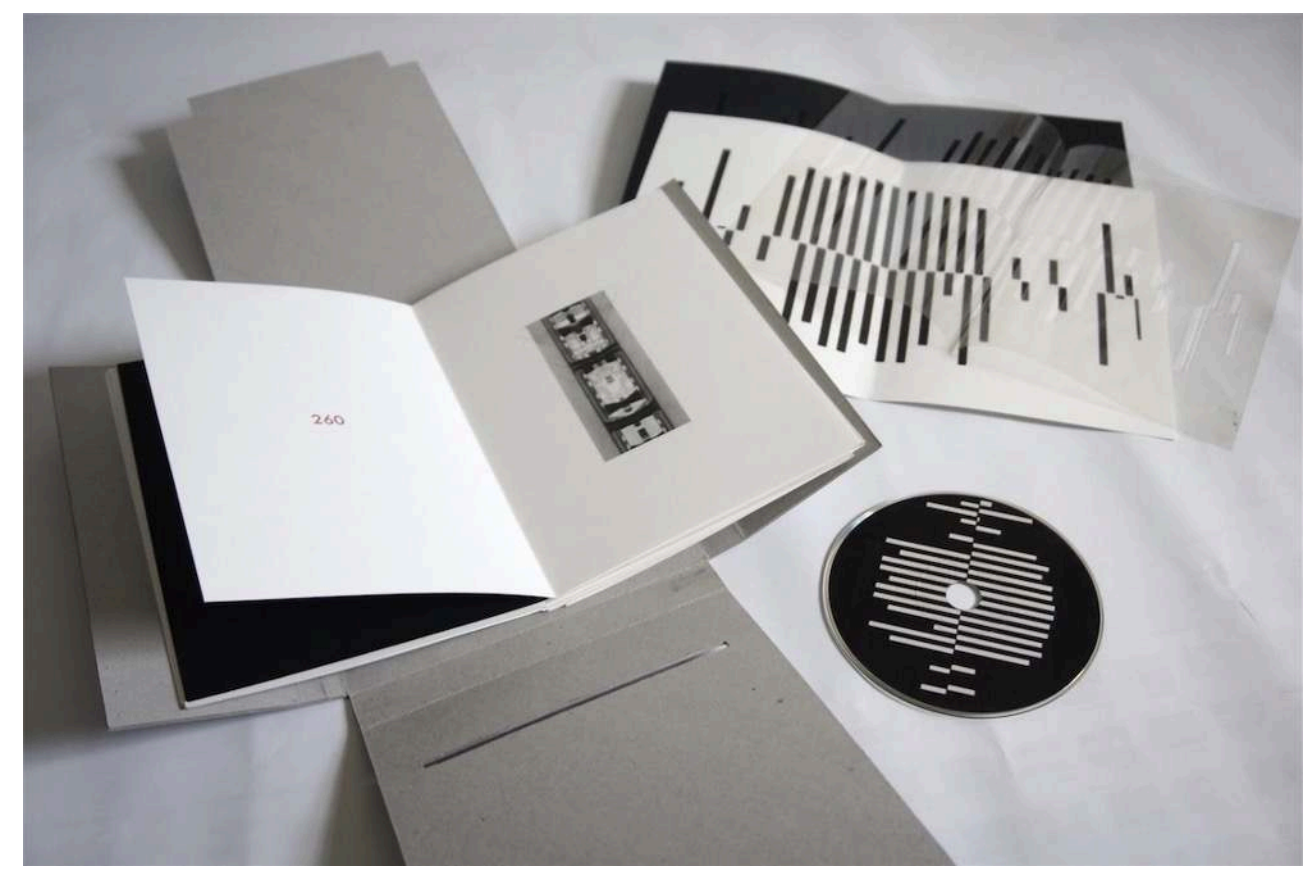

(c) Alexandre Melay.

vм : On voit dans ces expérimentations une intermedialité très forte et un dialogue entre art, architecture, musique, cinéma, littérature qui rappelle les propositions des avant-gardes soucieuses de repenser la mimesis et la place de l'art et de l'artiste dans la société. On observe également que le livre devient un espace d'exposition, de construction, de représentation et d'expérience autonome et interactive. En ce sens, vos œuvres allient de manière très efficace tradition (japonaise) et hyper-modernité.

AM : Pour conclure, je pense que cette sélection de livres d'artiste en tant qu'espaces alternatifs, multiples, autonomes et phénoménologiques permet une perpétuelle recherche de nouveaux formats. Les formes variées que j'ai utilisées dans mes créations donnent à l'objet-livre la possibilité de dépasser les contraintes d'une structure établie pour le reconfigurer en fonction d'un contenu dans une conception spatiale nouvelle. Même si mes objets-livres servent à des exercices artistiques et conceptuels, à travers les matières, les images, les figures, les traces qui s'offrent comme des tremplins pour l'esprit, ils sont aussi à considérer comme de véritables collections d'œuvres d'art en devenant un "lieu d'exposition”, comme un musée de papier : un véritable dispositif scénique inédit.

\section{RÉSUMÉS}

Le présent article s'inscrit dans une perspective de recherche-création interrogeant la question du format à travers le médium du livre d'artiste. Doté d'une personnalité matérielle et formelle, l'objet-livre est un support artistique ouvert favorisant les formats hybrides. C'est un objet tridimensionnel, assurément sculptural, pouvant prendre des formes multiples à travers des « 
systèmes » de formats pluriels : le « livre-accordéon », le « livre-édifice » ou encore le « livresonore " pour ne citer qu'eux. Ces nouvelles inventions formelles ont une dimension phénoménologique puisqu'ils génèrent une nouvelle relation spatiale entre l'objet et notre corps ou regard et sollicitent activement le lecteur/spectateur. L'objet-livre joue avec l'espace et les domaines artistiques, croisant et revisitant les conventions du dessin, de la sculpture et de l'architecture et mobilisant plusieurs sens.

This article is part of a research-creation project where the concept of "format" can be questioned through the medium of the artist's book. Endowed with specific material and formal qualities, the book-object is an open artistic medium generating hybrid formats; a threedimensional - sculptural - object with multiple shapes: "systems" of formats such as the "accordion-book", the "building-book" or even the "sound-book". These formal inventions create new phenomenological experiences as our physical presence is called upon in a movement including the gaze and the body. The book-object with its singular shapes and its unconventional "formats", becomes a sculpture, a piece of architecture, an exhibition space constructing a new spatiality and mobilising various senses.

\section{INDEX}

Mots-clés : livre d'artiste, objet-livre, œuvre-livre, systèmes, construction, formes, formats, trois dimensions, espace, spatialités, exposition

Keywords : artist's book, book-object, bookwork, systems, construction, shapes, formats, three dimensions, space, spatialities, exhibition

\section{AUTEURS}

\section{ALEXANDRE MELAY}

Artiste plasticien diplômé de l'École Nationale Supérieure des Beaux-Arts de Lyon et du Master Édition d'art et livre d'artiste, Alexandre Melay est l'auteur d'une thèse sur les concepts de temporalité et de spatialité dans l'esthétique japonaise, effectuée lors de résidences de créationrecherche au Japon à l'École française d'Extrême-Orient de Kyôto, ainsi qu'à la Maison FrancoJaponaise de Tôkyô/CNRS-MAEE. En tant que créateur de livres d'artiste, ses recherchescréations interrogent notamment les formes et les pratiques de l'édition, de l'imprimé et de l'objet-livre dans la création plastique contemporaine en lien avec l'écriture et la photographie, l'esthétique et la pensée japonaises. www.alexandremelay.com

\section{VALÉRIE MORISSON}

Université de Bourgogne

Valérie Morisson, Maître de Conférences en anglais à l'Université de Bourgogne, Dijon, est l'auteure d'une thèse portant sur l'art irlandais contemporain et ses rapports à l'identité nationale. Elle a publié plusieurs articles relatifs à la culture visuelle irlandaise (arts visuels, photographie et illustration, bande-dessinée) mettant en exergue le passage du nationalisme au postnationalisme culturel. Ses recherches sur la photographie contemporaine irlandaise et sur l'œuvre d'artistes féministes montrent que l'art et les pratiques artistiques interrogent l'histoire, la mémoire et les pouvoirs de l'image au sein de sociétés en constante mutation. Ses recherches actuelles se concentrent sur l'art contemporain, irlandais et britannique, et accordent une importance particulière au processus créatif. 